\title{
Rationale Bildgebung beim HWS-Trauma
}

Thomas Grieser

\section{Allgemeiner Teil}

Die Halswirbelsäule stellt aufgrund ihres morphologischen Aufbaus den beweglichsten Teil des gesamten Achsenskelettes dar. Diese hohe Beweglichkeit wiederum macht sie jedoch anfällig für Verletzungsmechanismen, die indirekt vor allem über Akzelerations- und Dezelerationskräfte ausgeübt werden. Aber auch direkte Krafteinwirkungen, z. B. beim Sturz auf den Kopf, gefährden in erster Linie die Halswirbelsäule, insbesondere ihren oberen Abschnitt. Obwohl nur ca. 2-3\% aller stumpfen Traumen zu HWS-Verletzungen führen, ist ihre frühzeitige Detektion wegen drohender Folgen wie Instabilität und neurologischen Defiziten immanent [1,2].

\section{Die Projektionsradiografie (HWS-Röntgen)}

Mit Ausnahme polytraumatisierter Patienten stellt die konventionelle Projektionsradiografie der HWS in 2 Ebenen sowie die Denszielaufnahme (ggf. ergänzt durch die
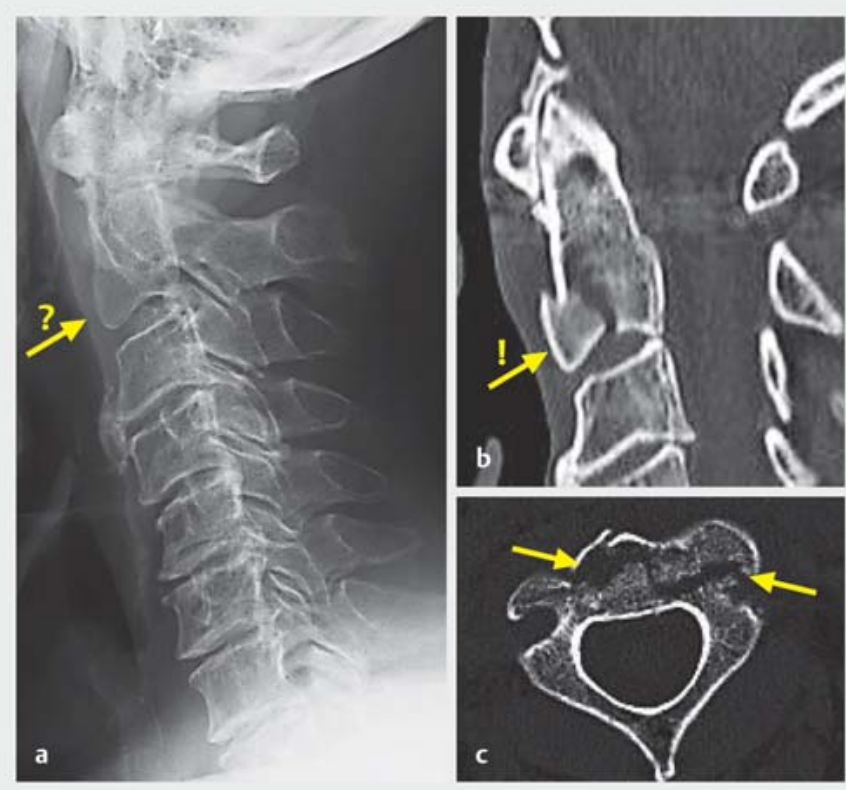

- Abb. 1 58-jähriger Patient, Treppensturz. a Röntgen am Unfalltag negativ, ebenso keine prävertebrale Weichteilschwellung (Pfeil). b Wegen persistierender Schmerzen nach 2 Wochen Wiedervorstellung: Teardropartige Fraktur an der Axisbasis (Pfeil). c Die Fraktur erstreckt sich quer durch die gesamte Axisbasis (Pfeile). sog. „Schwimmeraufnahme“ und Schrägprojektionen) nach wie vor den ersten bildgebenden Schritt in der diagnostischen Kette dar und wird vielerorts auch noch heute durchgängig so praktiziert. Prinzipiell ist hiergegen nichts einzuwenden, jedoch sollte man sich der großen diagnostischen Lücken durchaus bewusst sein: Die Sensitivität bez. relevanter zervikaler Frakturen beträgt bei der Projektionsradiografie lediglich $60-80 \%$ [2,3], d.h. die konventionelle Röntgendiagnostik übersieht in etwa $1 / 3$ der Fälle relevante HWS-Frakturen ( $\triangleright$ Abb. 1 und 2). Hinzu kommt, dass die röntgenologische Darstellung oftmals nicht die gesamte HWS suffizient und diagnostisch verwertbar abzubilden vermag; das gilt vor allem für den zervikothorakalen Übergang [4]. Daffner und Harris haben die sog. „ABC's“-Methode vorgeschlagen, nach der vorliegende HWS-Röntgenaufnahmen hinsichtlich sog. „footprints“, d.h. relevanter zervikaler Verletzungen (Alignmentstörungen, knöcherne [bony] Integrität, Gelenkstellungen [cartilage] und Weichteilauffälligkeiten [soft tissue]) beurteilt werden sollten [71].

Dieser diagnostischen Schwächen wegen findet das HWS-Röntgen in aktuellen Guidelines (American Association of Neurological Surgeons, 2013; [5]) nahezu keine Erwähnung mehr. Diesbezüglich haben aber bereits vor über 20 Jahren zwei bis heute gültige US-amerikanische bzw. kanadische Leitlinien Kriterien festgelegt, unter denen auf eine konventionelle Projektionsradiografie bei der Abklärung zervikaler Verletzungsfolgen verzichtet werden kann: die NEXUS- (National Emergency X-Radiography Utilization Study) und die Canadian C-Spine-RuleStudie (CCSR) [6, 7]. Spätere Studien wiesen auf niedrigere Sensitivitäten und Spezifitäten der NEXUS-Kriterien hin [8] sowie darauf, dass sich diese Kriterien bei älteren Patienten mit HWS-Trauma als insuffizient erwiesen [9], wohingegen sich die CCSR aufgrund ihrer hohen Sensitivität (>90\%) und exzellenten Interobserver-Übereinstimmung als überlegen erwiesen hat [81].

\section{Funktionsaufnahmen in Flexion und Hyperextension}

Sie haben im akuten Traumasetting keinen Stellenwert, wenn sie nicht gar kontraindiziert sind. Zudem bleibt ihre diagnostische Aussagekraft zu diesem Zeitpunkt vernachlässigbar gering. Bei der chronischen zervikalen Instabilität hingegen - sei sie traumatisch oder entzündlich verursacht oder degenerativ bedingt - ist sie durchaus von Nutzen: Dies gilt insbesondere für den Nachweis 


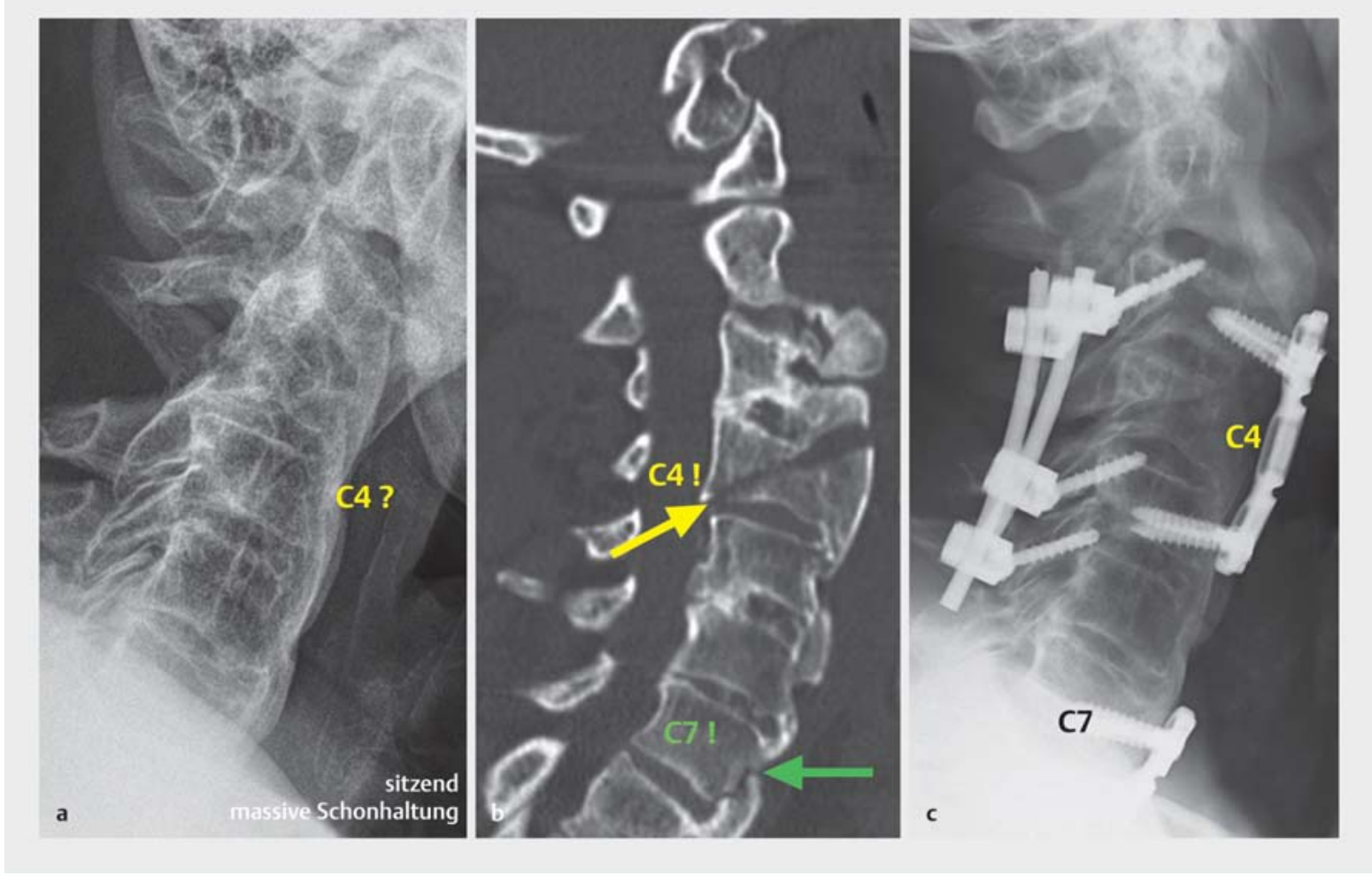

Abb. 2 66-jähriger Patient, unbeobachteter häuslicher Sturz, Kopfplatzwunde. a Seitliches Röntgenbild nicht diagnostisch! b CT zeigt die transvertebrale Fraktur HWK IV bei DISH, aber auch Spondylophytenfraktur bei HWK VII/BWK I. c Z. n. 2-EtagenSpondylodeseversorgung. (DISH: diffuse idiopathische Skeletthyperostose/Morbus Forrestier).

atlantoaxialer Instabilitäten ( $\vee$ Abb. 3) und instabiler Denspseudarthrosen ( $\bullet$ Abb.4). Subaxiale segmentale Instabilitäten sind meist Folge nicht ausgeheilter diskoligamentärer Verletzungen, sind aber meist geringer ausgeprägt als die atlantoaxialen Dislokationen. Vor dem unkritischen Einsatz von Funktionsaufnahmen sei jedoch gewarnt, was insbesondere für Patienten gilt, die bereits in Ruhe und bei normalen Kopfbewegungen Schmerzen angeben. Falls diagnostisch erforderlich, würden sich für solche Fälle eventuell ärztlich geführte Flexions-/Hyperextensionsmanöver unter fluoroskopischer Sicht anbieten.

\section{Computertomografie}

Die CT der HWS stellt mittlerweile die wichtigste, da - für knöcherne Verletzungen - spezifischste radiologische Abklärungsmethode dar und überwindet die o.g. diagnostischen Nachteile der Projektionsradiografie [10]. Dies gilt insbesondere seit der flächendeckenden Verfügbarkeit der Mehrzeilen-CT, welche die einfachen, aber diagnostisch überaus wertvollen multiplanaren Rekonstruktionen (MPR) in sagittaler und koronaler Ebene erlaubt. Zahlreiche Studien haben eindrucksvoll die hohe diagnostische Sensitivität und Spezifität der CT zur Abklärung knöcherner HWS-Verletzungen zeigen können [11 13].

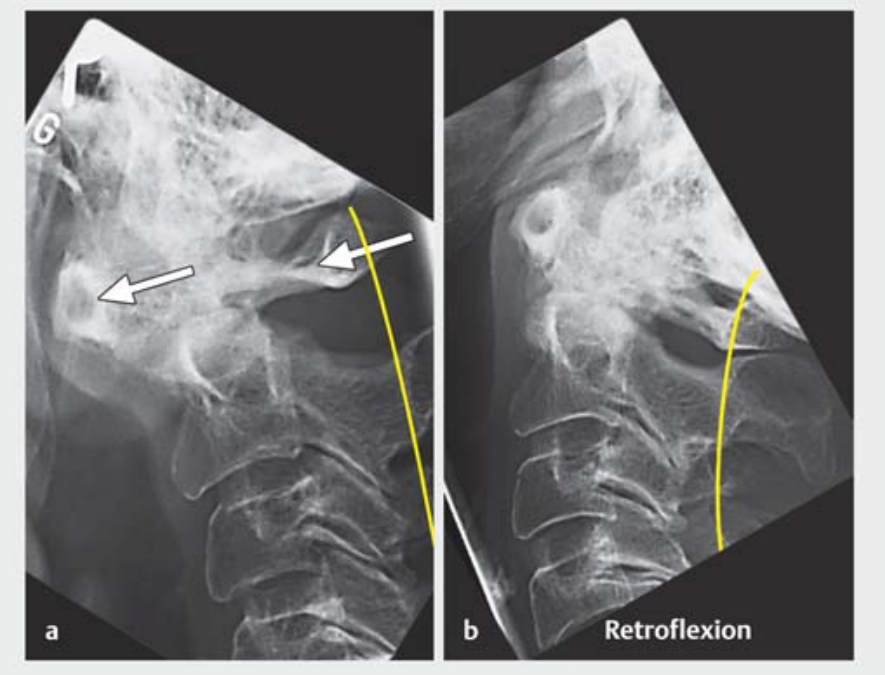

Abb. 3 Atlantoaxiale Instabilität: 68-jährige Patientin, bekannte langjährige Rheumatoidarthritis, jetzt zunehmende HWS-Beschwerden. a In Inklination deutliche atlantoaxiale Dislokation nach ventral (Pfeile); spinolaminäre Linie unterbrochen (gelbe Linie). b In Reklination erfolgt eine vollständige Reposition (spinolaminäre Linie korrekt). 

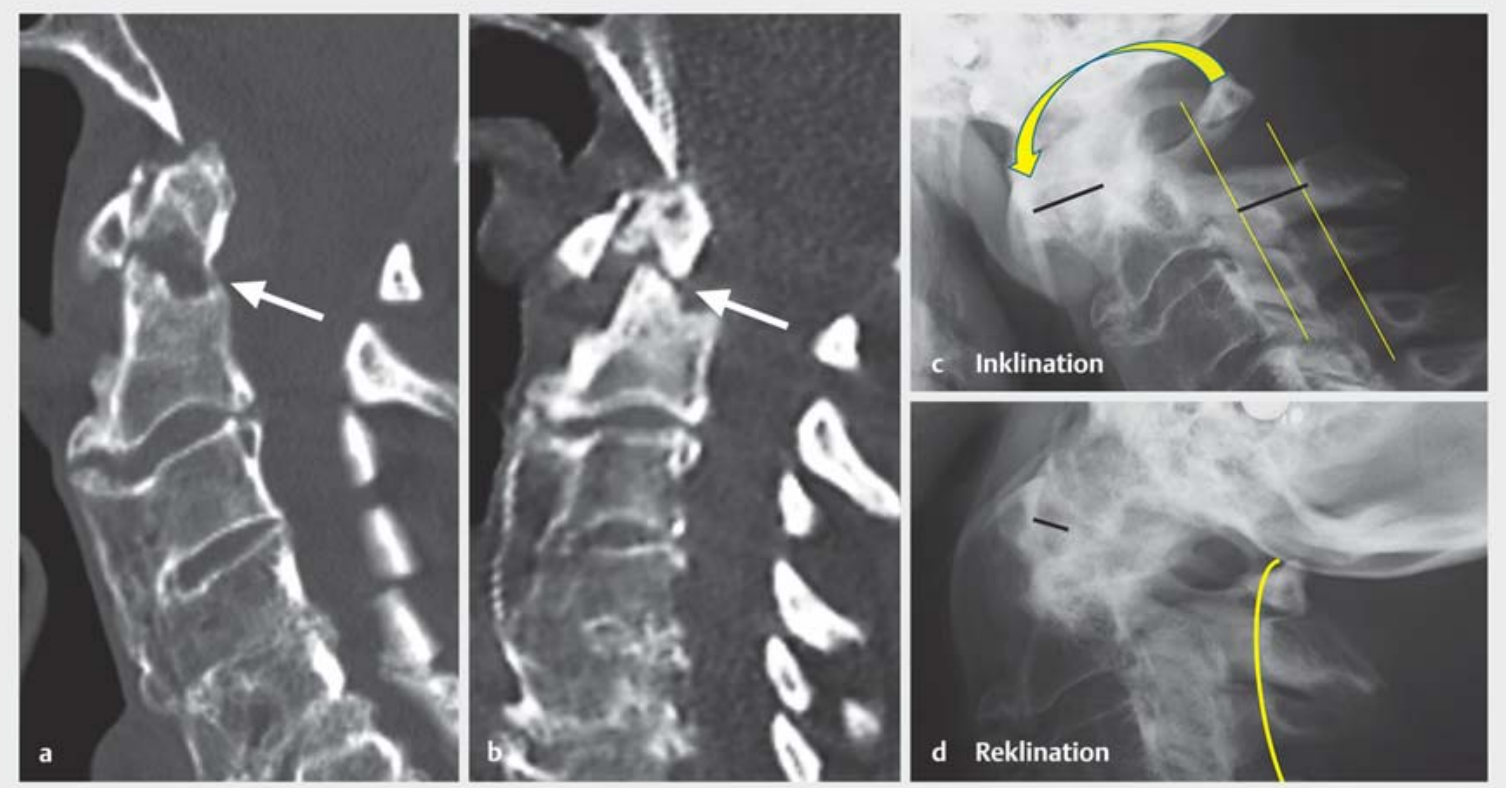

- Abb. 4 71-jähriger Patient: a Bekannte Densfraktur unbekannten Alters (Pfeil). b 1,5 Jahre später: anteriore atlantodentale Dislokation und Denspseudarthrose (Pfeil). c + d die Funktionsaufnahmen zeigen die deutliche anteriore Dislozierbarkeit des Atlas (gebogener Pfeil): instabile Denspseudarthrose.
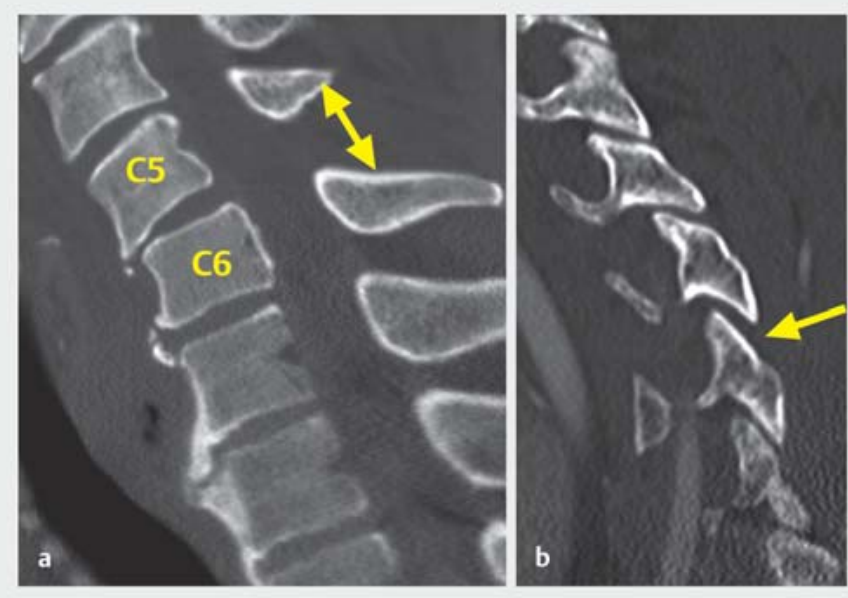

- Abb. 5 a Monosegmentale Erweiterung des Interspinalraumes (Doppelpfeil) mit Angulation von HWK V gegenüber HWK VI. b Partielle Entdeckelung der Facetten ohne Luxation (Pfeil): Intraoperativ ergab sich eine vollständige diskoligamentäre Läsion. Quelle: Grieser T, Fessl R. Wirbelsäule. In: Bohndorf K, Imhof H, Wörtler K. Radiologische Diagnostik der Knochen und Gelenke. 4. Aufl. Stuttgart: Thieme; 2017: 79-105.

Mittlerweile steht es außer Frage, dass die CT der HWS beim polytraumatisierten Patienten (z. B. Hochrasanztrauma beim Frontalcrash) obligat ist, um akut versorgungspflichtige knöcherne Verletzungen zu identifizieren (zum Einsatz der MRT siehe weiter unten).
Jedoch sollte nicht übersehen werden, dass auch sog. Bagatelltraumen, wie z. B. der Sturz aus „innerer Ursache“ (plötzliche Bewusstlosigkeit aus kardialer, zerebraler oder sonstiger Ursache), mit Anprall des Kopfes gegen nahe stehende Tisch-, Bett- oder Heizkörperkanten infolge des völligen Versagens muskulärer Schutzreflexe zu schweren HWS-Verletzungen führen können, die bei alleiniger Fokussierung auf mögliche intrakranielle Verletzungsfolgen (z. B. intrazerebrale Blutung [ICB]) ohne Weiteres übersehen werden können.

Wie bereits beim konventionellen Röntgen bedarf auch die CT einer subtilen Durchmusterung des sagittalen (und koronalen) Alignments. Zwar gibt die Literatur hinsichtlich signifikanter Alignment-Abweichungen einige wertvolle Kenngrößen an (>11 ${ }^{\circ}$ Angulation, $>3,5 \mathrm{~mm}$ Translation, $>50 \%$ Facettenabdeckelung etc. $[14,15])$, die aber im Einzelfall kritisch analysiert werden müssen. Nach eigener Erfahrung muss „sprunghaften“, monosegmentalen Alignment-Störungen große Aufmerksamkeit zuteil werden: Facettengelenksdislokationen sowie -verhakungen (sog. Interlocking), auffälliges Fehlen der gegenseitigen Facettenüberdachungen oder das Klaffen der facettären Gelenkflächen bzw. Frakturen der Gelenkfacetten ( $\bullet$ Abb. $\mathbf{5})$.

Darüber hinaus weisen selbst kleine knöcherne Fragmente an den HWK-Vorderkanten auf relevante Avulsionsverletzungen und auf zugrunde liegende diskoligamentäre Zerreißungen hin (Teardrop-Verletzungen - siehe weiter unten). Diese würden in jedem Falle eine weitere MRT- 
Abklärung nach sich ziehen, da es sich zumeist um Hyperflexions- bzw. Hyperextensions-Distraktions-Verletzungen (sog. B-Verletzungen nach AO) handelt.

Aufgrund der hohen HWS-Mobilität sind zervikale Luxationen bzw. Luxationsfrakturen häufiger anzutreffen. Ihre Detektion gelingt zwar leicht, allerdings erfordert die damit einhergehende Myelongefährdung große Aufmerksamkeit und notfallmäßige MRT-Abklärung (nach Möglichkeit noch präoperativ; • Abb. 6).

Eine besondere diagnostische Herausforderung stellen Rotationsverletzungen der HWS, sog. C-Verletzungen nach AO, dar. Aufgrund oftmals zwischenzeitlich und „spontan“ erfolgter „Repositionen“ im Stiff Neck oder Ausrichtung auf dem Spine Board fehlt mitunter die offensichtliche Rotationsfehlstellung; umso mehr sollte nach indirekten Zeichen eines rotatorischen Verletzungsmusters gefahndet werden (asymm. Frakturen der Facettengelenke, der Querfortsätze, aber auch der Pedikel sowie der oberen Rippenhälse). Diese Verletzungsmuster sind praktisch dafür beweisend, dass zumindest eine relevante rotatorische Kraftkomponente während des Traumas eingewirkt haben muss ( $\triangleright$ Abb. 7). Diesbezüglich konnte gezeigt werden, dass z. B. ein konservatives, nicht operatives Management einseitiger unverschobener $\mathrm{Fa}$ cettenfrakturen ein schlechteres klinisches Outcome zeigten [18].

Die Myelografie ( $\bullet$ Abb. 8) - als zervikale oder PanmyeloCT durchgeführt mit anschließender multiplanarer Reformation - hat zwar ihren Stellenwert gegenüber der MRT (einschließlich der 2-D- bzw. 3-D-MR-Myelosequenzen) eingebüßt, dennoch kann ihre gute intraspinale Ortsauflösung zum Nachweis therapiepflichtiger medullärer oder auch radikulärer Kompressionszustände genutzt werden, wenngleich eine direkte Myelonbeurteilung nicht möglich ist. Ein solches Verfahren ist immer dann zu bedenken, wenn eine MRT nicht möglich, kontraindiziert und schlicht nicht verfügbar ist ( $\bullet$ Abb. 9).

\section{Magnetresonanztomografie}

Die MRT spielt zwar in der modernen Traumadiagnostik eine bedeutende und noch weiter zunehmende Rolle, jedoch besitzt sie in der täglichen Routine und im Work-up polytraumatisierter Patienten gegenüber der CT eine klar nachgeordnetete Priorität. Vordringlich allerdings wird eine MRT-Abklärung immer dann, wenn neurologische Funktionsdefizite bis hin zur hohen Querschnittsymptomatik erkannt werden bzw. sogar im Vordergrund stehen [20].

Dies gilt auch - was häufig unterschätzt wird - für Unfälle und Traumen bei degenerativ vorgeschädigter oder ankylosierter HWS, da selbst geringe oder als nur gering angesehene Traumen in solchen Fällen zu Myelonkompressionsverletzungen infolge einer vorbestehenden de-
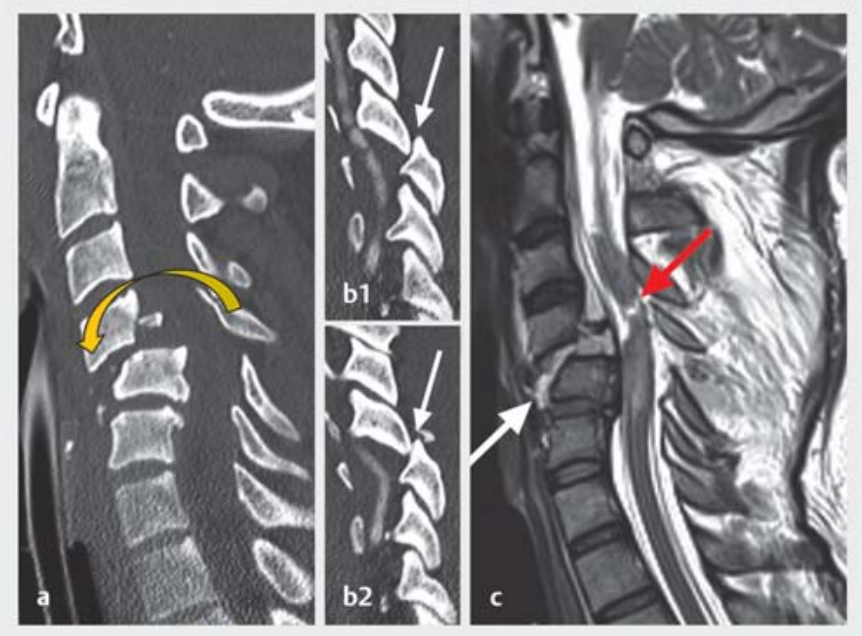

- Abb. 6 Subaxiale Luxationsfraktur HWK IV/V: gebogener Pfeil in a zeigt eine schwere Alignmentstörung mit vollständiger diskoligamentärer Zerreißung (dicker Pfeil in c) und bilateral verhakter Facettenluxation (dünne Pfeile in b1 + b2) sowie Myelontranssektion (roter Pfeil in c).
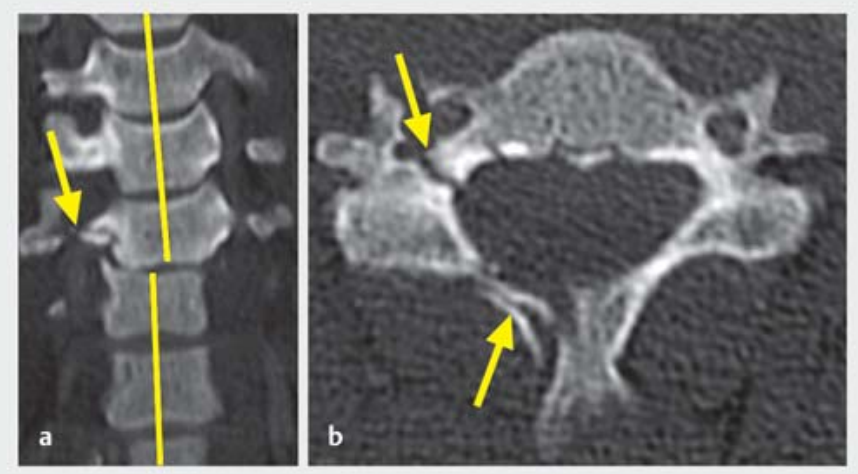

- Abb. 7 Rotationsverletzung: 45-jährige Frau, Reitunfall. a „Windschiefes" frontales HWS-Alignment mit Versatz im Segment HWK V/VI; b Einseitige Frakturen (Pfeile) als Hinweis auf eine Rotationsverletzung.

generativ bedingten Spinalkanalenge führen können $[16,17]$ ( $\bullet$ Abb. 10).

Neben der akuten Myelonabklärung spielt die MRT beim Nachweis diskoligamentärer Verletzungen eine entscheidende Rolle. Auch wenn diese Läsionen in der Mehrzahl keine Akutindikation darstellen, so kommt ihnen prognostisch und therapeutisch eine entscheidende Bedeutung zu. So erfordern diskoligamentäre Rupturen i.d.R. eine stabilisierende Spondylodeseversorgung mit oder ohne Bandscheibenersatz. 

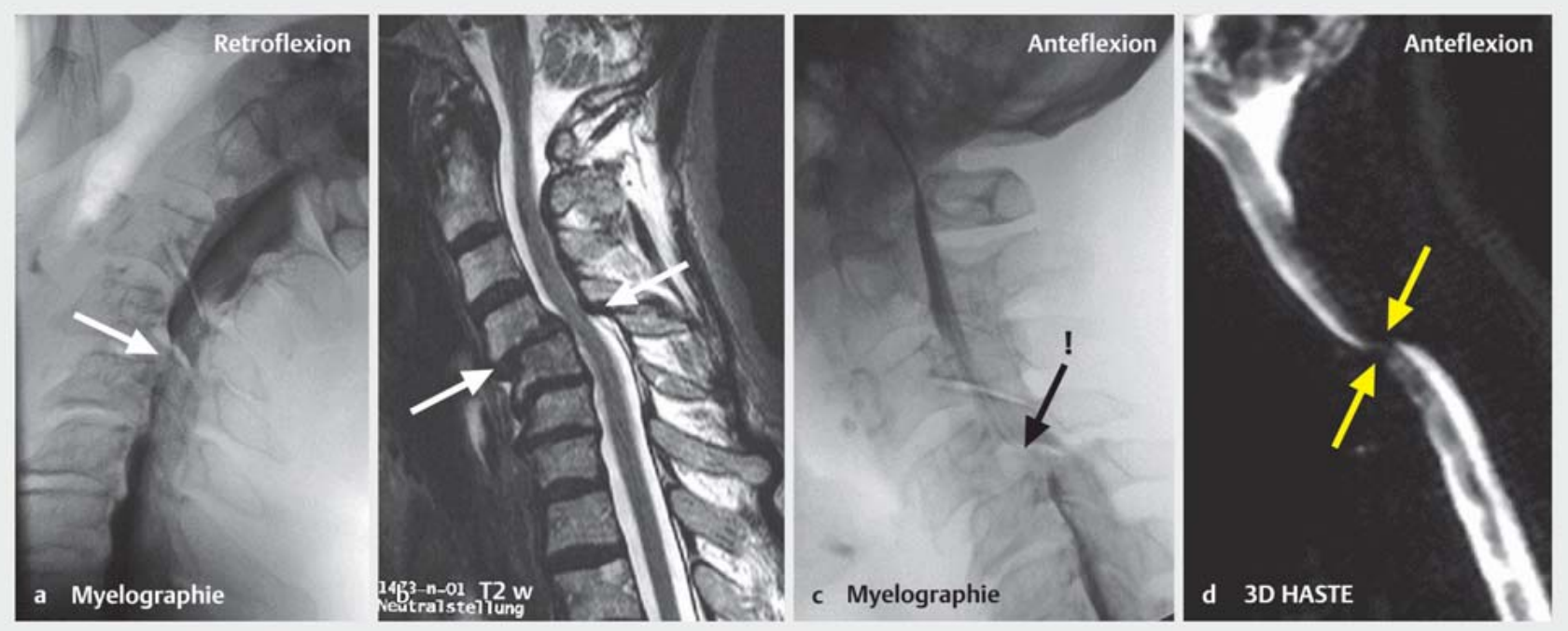

- Abb. 8 Chronische, atraumatische segmentale Hypermobilität auf Niveau HWK IV/V: a In Reklinationsstellung freie intrathekale KM-Passage (Pfeil). b In Neutralstellung (liegend) segmentale Ventrolisthese mit Myelonbedrängung (Pfeile); c Kompletter Liquorstopp in Anteflexion (Pfeil); d MR-Funktionsdiagnostik: vollständige Okklusion des Subarachnoidalraumes in Inklinationshaltung (Pfeile). Quelle: Prof. Dr. A. Berlis, Augsburg.

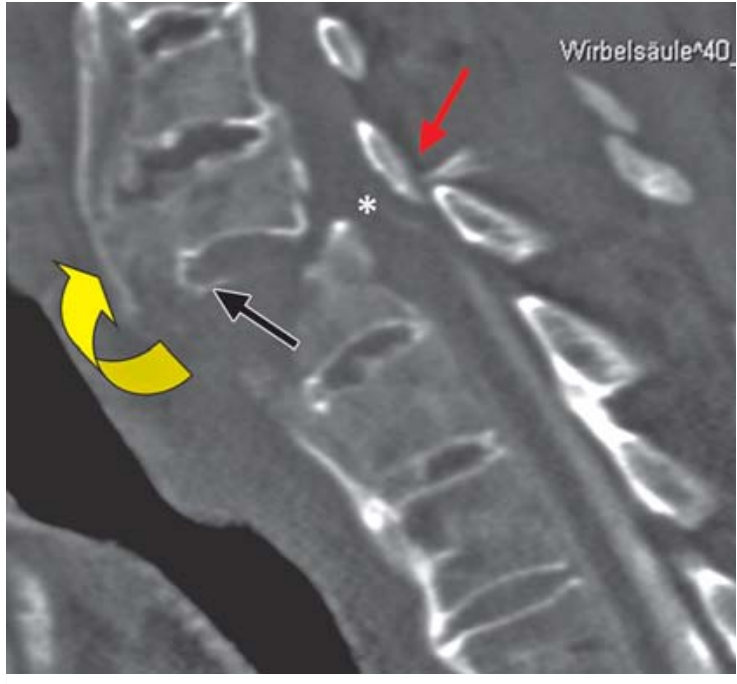

- Abb. 9 CT-Myelografie bei Hyperextensionsfraktur HWK IV/V (gelber Pfeil): bilaterale Facettenluxation (roter Pfeil; Luxation nicht gezeigt) mit Zereißung der vorderen Säule einschl. der hyperostotischen Spondylose mitsamt einem Vorderoberkantenfragment (schwarzer Pfeil), zugleich ventrale Spondylolisthesis mit kneifzangenartiger Myelonkompression (*): klinisch bestand eine Tetraparese. Beachte den Abbruch der kontrastierten Liquorsäule auf Luxationsniveau. Quelle: Grieser T, Fessl R. Wirbelsäule. In: Bohndorf K, Imhof H, Wörtler K. Radiologische Diagnostik der Knochen und Gelenke. 4. Aufl. Stuttgart: Thieme; 2017: 79-105.
Das diagnostische Problem besteht vielfach darin, die Signalveränderungen innerhalb des diskoligametären Komplexes richtig zu deuten bzw. zu interpretieren. Neben den rein kontusionell bedingten intradiskalen Signalerhöhungen in wassergewichteten Sequenzen bedürfen fokale Signaländerungen innerhalb des Anulus fibrosus und der Längsbänder besonderer kritischer Aufmerksamkeit und Beachtung. So ist es nicht immer eindeutig möglich, vermeintliche oder tatsächliche diskoligamentäre Signalalterationen klar als Ruptur - oder eben Nichtruptur - zu definieren ( $\bullet$ Abb. 11) [19]. Im Unterschied hierzu gelingt die Beurteilung des posterioren Bandapparates (Ligg. flava, interspinosa et supraspinale) sicher und zuverlässiger, was somit die Einschätzung der Intaktheit der dorsalen ligamentären Zuggurtung recht zuverlässig erlaubt bzw. ermöglicht [21] ( $\mathbf{A b b}$. 12). Zwar konnte eine aktuelle prospektive Multicenterstudie nachweisen, dass mittels MRT in bis zu 23,6\% zusätzliche Befunde im Vergleich zum CT erhoben werden konnten, deren tatsächliche Therapierelevanz wurde aber nicht evaluiert [22]. Insofern muss hier die erst jüngst in einer weiteren Studie festgestellte, hohe Rate falsch positiver Befunde dagegengehalten werden, was das Problem einer unnötigen (operativen) „Übertherapie“ evoziert [23].

Ein weiterer wichtiger Teilaspekt in der Evaluierung zervikaler Verletzungen und ihrer Folgen stellt die Beurteilung der Vertebralarterien hinsichtlich der Detektion von Dissektionen dar ( $\bullet$ Abb. 13a,b). Dieses Problem wird in der Praxis eher unterschätzt [24]. Sofern bereits eine kontrastmittelunterstützte CT-Angiografie der Halsgefäße im Rahmen der Polytraumaabklärung erfolgte, kann eine Vertebralisdissektion schon primär relativ sicher erkannt 

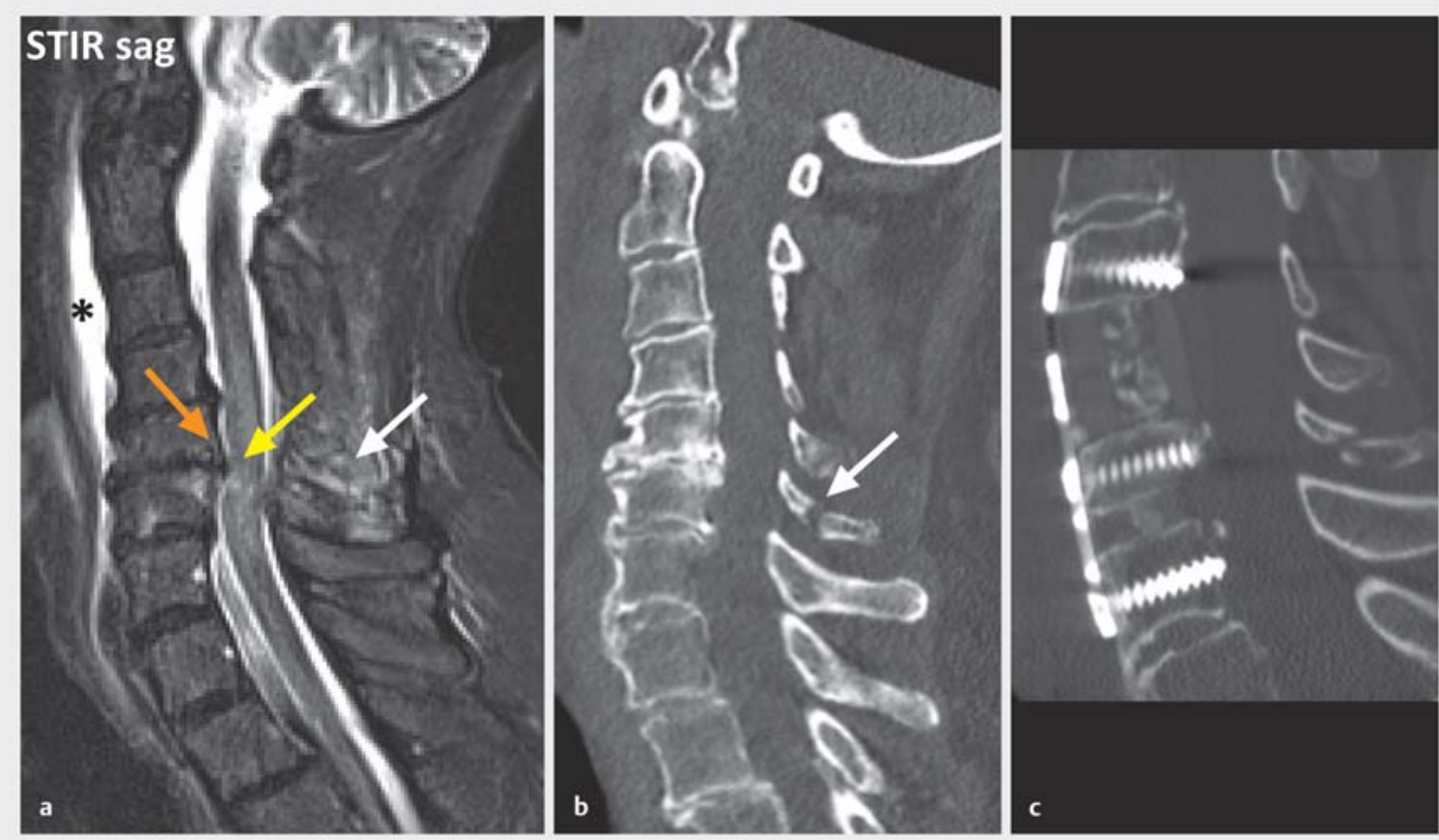

- Abb. 10 63-jähriger Patient, Sturz von der Leiter, progrediente Tetraparese. a Verletzung der dorsalen Zuggurtung (weißer Pfeil), Myelonkontusion (gelber Pfeil) und vorbestehende degenerative Spinalkanalstenose bei HWK V/VI (orangefarbener Pfeil), zusätzlich prävertebrales Hämatom ( ${ }^{*}$ ). b CT zeigt zusätzlich die Dornfortsatzfraktur (Pfeil). c Z. n. ventraler Spondylodese und HWK-V-Ersatz.
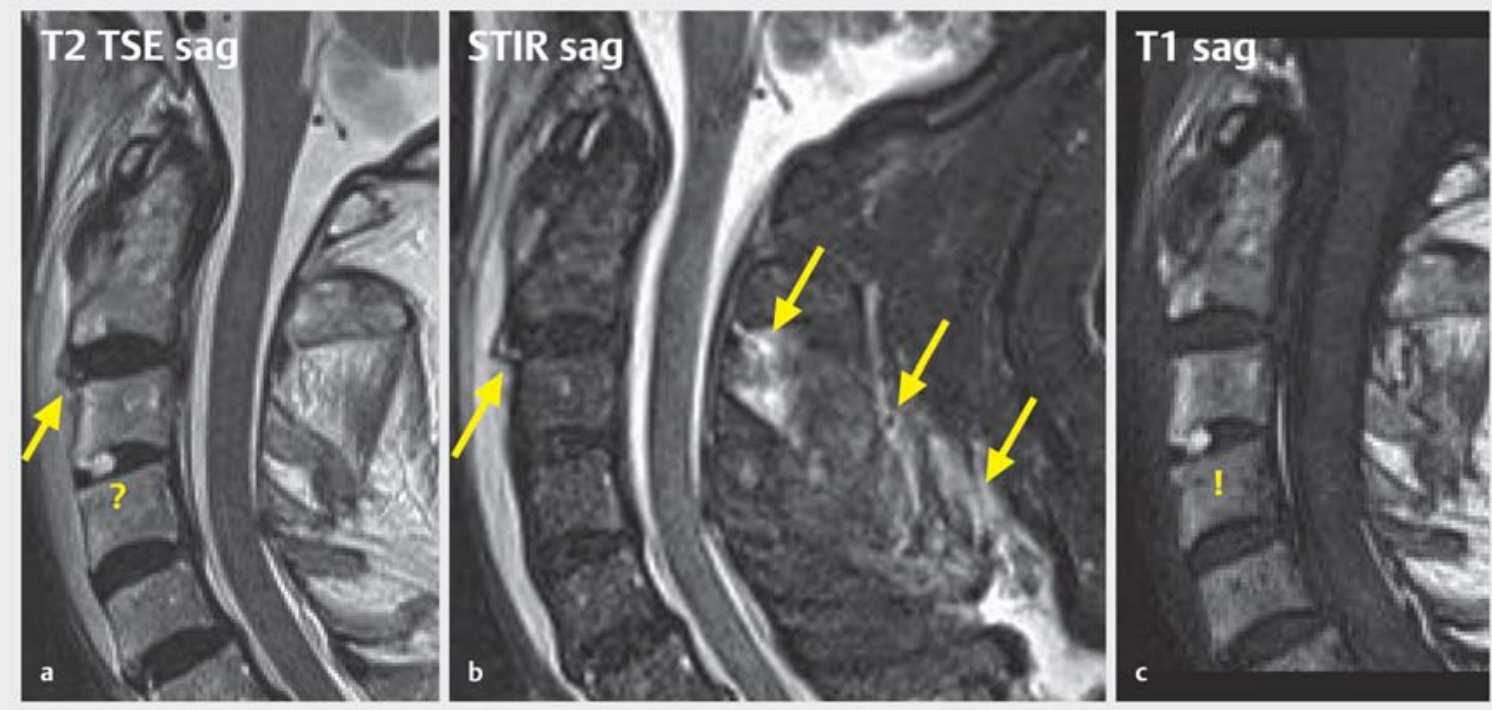

- Abb. 11 Falsch positiver Befund im Segment HWK II/III: a Verdacht auf Längsbandruptur (Pfeil), zusätzlich patholog. Signal in C III/IV (?). b Langstreckige dorsale Bandzerreißung (gelbe Pfeile). c „Pathologisches“ Signal in C III/IV $\rightarrow$ fokale Verfettung bzw. Verknöcherung (!). Intraoperativ erwiesen sich sowohl das vordere Längsband als auch der anteriore Anulus fibrosus auf Höhe HWK II/III als intakt. 

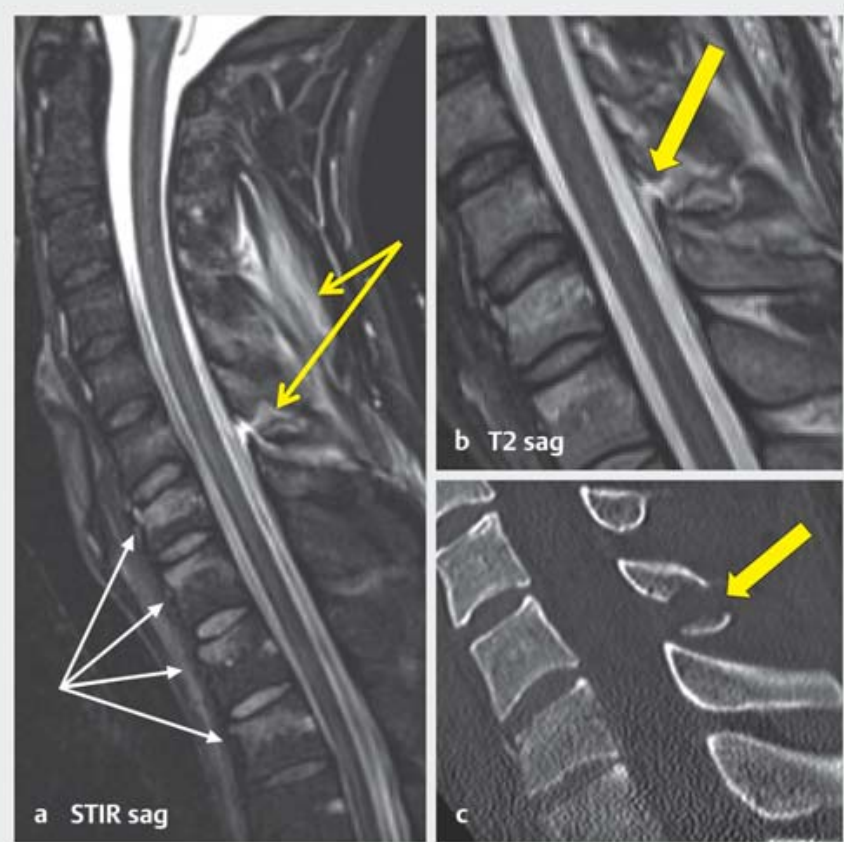

- Abb. 12 28-jähriger Mann, Fahrradsturz. a Nuchales interspinöses Weichteilödem mit Lücke im Bereich der Ligg. flava (gelbe Pfeile); zusätzlich obere thorakale Flexions-Kompressions-Frakturen (schmale Pfeile). b Verdeutlichung der segmentalen Ligg.-flava-Ruptur (Pfeil). c Zusätzlich Darstellung der Processus-spinosus-Fraktur (Pfeil).
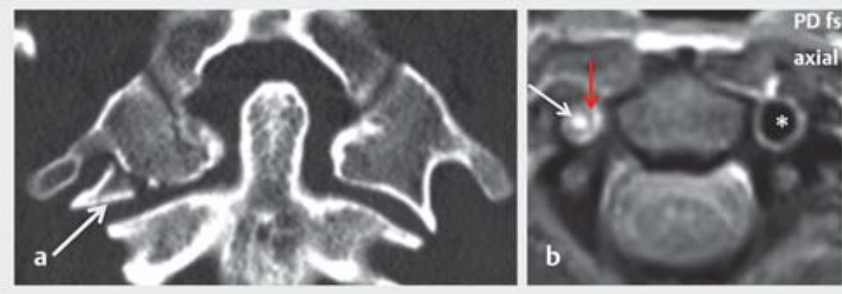

- Abb. 13 a Junge Frau, Sturz vom Pferd: Initiale CT-Abklärung ergab eine Gelenksockelfraktur des Atlas rechts (Pfeil), im Verlauf fluktuierende neurologische Symptomatik. b Ipsilaterale traumatische Vertebralisdissektion: wahres Lumen (weißer Pfeil) mit langsamem Fluss (DD Verschluss); Dissektionsmembran und falsches Lumen (roter Pfeil); regelrechtes flussbedingtes Flow-void $\left({ }^{*}\right)$ links.

bzw. ausgeschlossen werden. Jedoch sind es mitunter ungewöhnliche Verletzungsmechanismen („Einrenkungen“ im Rahmen einer Manualtherapie, Kampfsport wie Judo und Ringen, forcierte Kopfdreh- und -wendebewegungen), die initial nicht an eine Vertebralisdissektion denken lassen [25]. Erst eine fortgesetzte oder neu entstandene klinisch-neurologische Symptomatik führt dann zu einer weiterführenden Diagnostik mittels MRT, welche dann - bei angepasstem geeignetem Untersuchungsprotokoll - die Frage nach einer Dissektion sicher beantworten kann. Ähnliches gilt für die - ebenfalls selten zu beobachtende - traumatische Karotisdissektion [26].

\section{Spezieller Teil}

\section{Die okzipitoatlantoaxiale Region}

Verletzungen, die den Bereich der Schädelbasis (Okziput) bis zum Axis betreffen, bilden einen gesondert zu besprechenden Abschnitt, während Verletzungen, die sich unterhalb HWK II ereignen, als sog. subaxiale HWS-Läsionen bereits denen der übrigen WS-Abschnitte ähneln oder mit diesen identisch sind.

Der kraniozervikale Übergang ist einerseits ein hochmobiler Abschnitt (ca. 50\% der zervikalen Gesamtflexion/-extension und der Gesamtrotation entspringen diesem Segment; [27]), andererseits sichern im Wesentlichen die kräftigen kapsuloligamentären Strukturen die Stabilität dieser Region ab, was sie anfällig für akute und chronisch-persistierende kraniozervikale Instabilitäten macht.

\section{Okziput und kraniozervikaler Übergang}

Sowohl axiale als auch schräg einwirkende Kräfte gegen den Kopf können die okzipitale Schädelbasis treffen und zur Frakturen führen, die sowohl das Foramen magnum als auch die Okzipitalkondylen betreffen (vgl. Einteilung n. Anderson und Montesano, Typen I-III, 1988; [28]). Während Schädelbasisfrakturen (Typ II, stabil) noch recht sicher in der CT diagnostiziert werden, werden Impressionsfrakturen der Okzipitalkondylen (Typ I, zumeist stabil) häufig und leicht übersehen ( $\vee$ Abb. 14). Besonderes Augenmerk erfordert die Würdigung knöcherner Avulsionsfrakturen der Ligg. alaria (Typ III, instabil): Sie sind Folge hochrasanter Akzelerations-/Dezelerationstraumen und haben i.d.R. schwere obere Halsmarkschäden, häufig mit hoher Querschnittsymptomatik, zur Folge oder enden tödlich [29]. Praktisch ist es wichtig zu wissen, dass derartige knöcherne Alariaausrisse relativ sicher im CT erkennbar sind ( $\bullet$ Abb. 15), während sie sich in der MRT oft genug nur schwer identifizieren lassen. Hierzu schlugen Tuli et al. 1997 eine MR-basierte Einteilung vor, wonach eine über $2 \mathrm{~mm}$ dislozierte Kondylenfraktur mit Nachweis einer ligamentären kraniozervikalen Zerreißung als instabil gilt (Typ IIB) [30].

\section{Okzipitoatlantale Dislokationen}

Hierbei handelt es sich zumeist um rein ligamentäre Zerreißungen zwischen Kopf und HWS. Obwohl es sich um schwere und schwerste Verletzungen handelt, wird ihr Ausmaß im Röntgen und auch in der CT regelhaft unterschätzt, da man ja kaum knöcherne Läsionen sieht [31]. Den Versuch einer ersten, noch recht simplen Einteilung nach der Dislokationsrichtung gaben Traynelis et al. 1986 

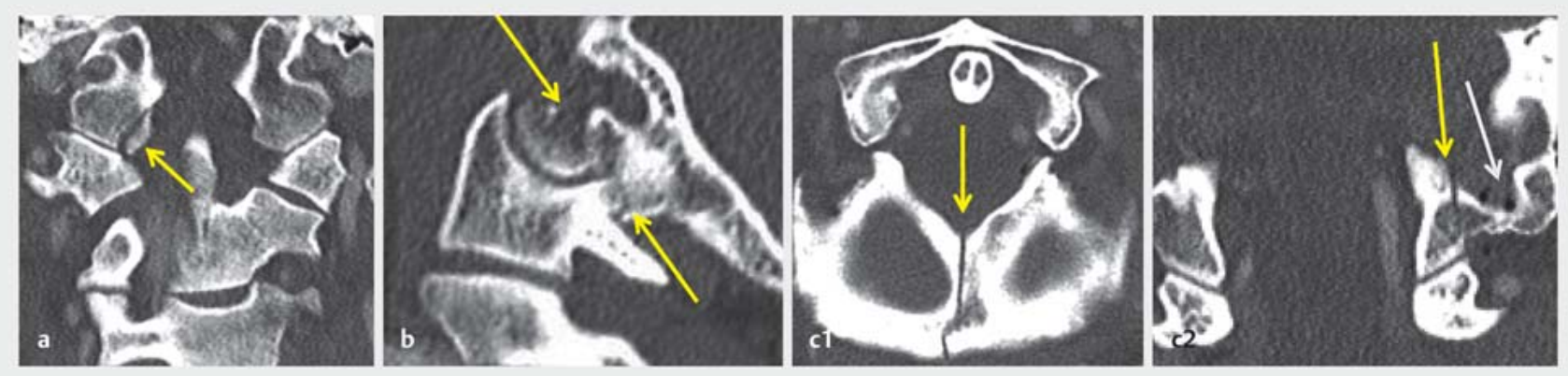

- Abb. 14 Drei Fälle okzipitaler Frakturen: a Innenseite Okzipitalkondyle (DD knöcherne Ligg.-alaria-Avulsion; Pfeil). b Okzipitale transkondyläre Scherfraktur (Pfeile). c Sagittale Schädelbasisfraktur (Pfeil in c1) und linksseitige Kondylusfraktur (gelber Pfeil; beachte Lufteinschlüsse in der Nähe des Sinus sigmoideus; weißer Pfeil in c2).

[87]. Hier können zwar die zahlreichen und erprobten Hilfslinien sowie Distanzmessungen helfen (z.B. die Harris-Methode; [74,75]; $\triangleright$ Abb. 16), jedoch sollten diese nur zur Orientierung und groben Abschätzung dienen; letztlich erfordern Schwere des Traumas und resultierende neurologische Ausfallssymptomatik eine rasche Abklärung des Weichteil- und Myelonschadens mittels der MRT. Darauf fußt letztlich die rezente Klassifikation (2007) nach Horn et al. [88].

An dieser Stelle sei ganz besonders auf die schwerwiegenden, oft auch tödlichen kindlichen Verletzungen des kraniozervikalen Überganges hingewiesen: Der im Vergleich zum übrigen kindlichen Körper relativ schwere Kopf und die noch recht schwach entwickelte Nackenmuskulatur bedingen zum einen hohe Trägheitsmomente und führen zum anderen zu enormen Translations- und Distraktionskräften an der ligamentären okzipitozervikalen Junktion, was in der Folge zu ihrer vollständigen Zerreißung führt mit den entsprechenden deletären Folgen für das obere Halsmark bzw. der vertebralen hinversorgenden Gefäße [32] ( $\bullet$ Abb. 17). Da diese schweren Verletzungen oftmals ohne jegliche röntgenologisch bzw. im CT fassbaren Frakturen auftreten (sehr wohl aber mit i. d. R. pathologischen kraniozervikalen Distanzen), folgen diese Traumapathologien dem sog. SCIWORA-Konzept (Spinal Cord Injury WithOut Radiographic Abnormality; [33] ( Abb. 18).

\section{Isolierte Atlasfrakturen}

Sie kommen in bis zu $13 \%$ aller akuten zervikalen Verletzungen vor und stellen aufgrund der Ringstruktur des Atlas eine Besonderheit dar [34]. Sie entstehen infolge eines axialen Krafteinwirkungsvektors oder einer erheblichen Hyperextension (mit oder ohne Rotation), die über die Okzipitalkondylen nach kaudal abgeleitet werden. Dabei wirken sowohl die schräg gestellten Okzipitalkondylen als auch die entgegengesetzt gestellten Gelenkfacetten des Axis geradezu als „Sprengkeile“ auf den Atlasring, der auseinandergerissen wird (Berstungsfrak-
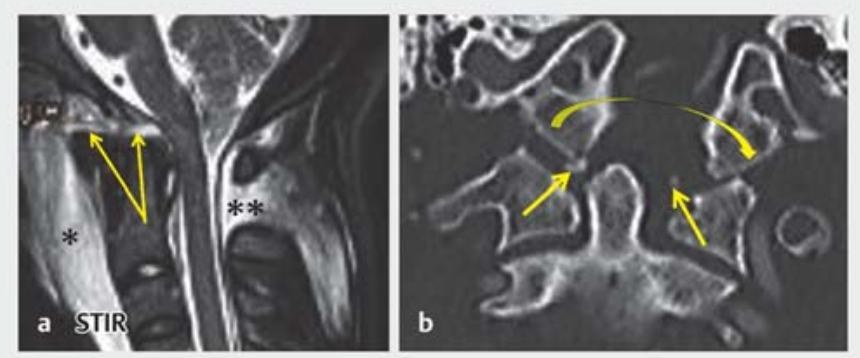

- Abb. 15 Beidseitige Ligg.-alaria-Ruptur. a Vollständige ligamentäre Zerreißung zwischen Basion und Dens (Pfeile), Ruptur der atlantoaxialen Bänder $\left({ }^{* *}\right)$ und großes prävertebrales Hämatom $\left({ }^{*}\right)$; b knöcherne Avulsionen der Ligg. alaria (Pfeile) mit querer Translation des Okziputs (gebogener Pfeil).

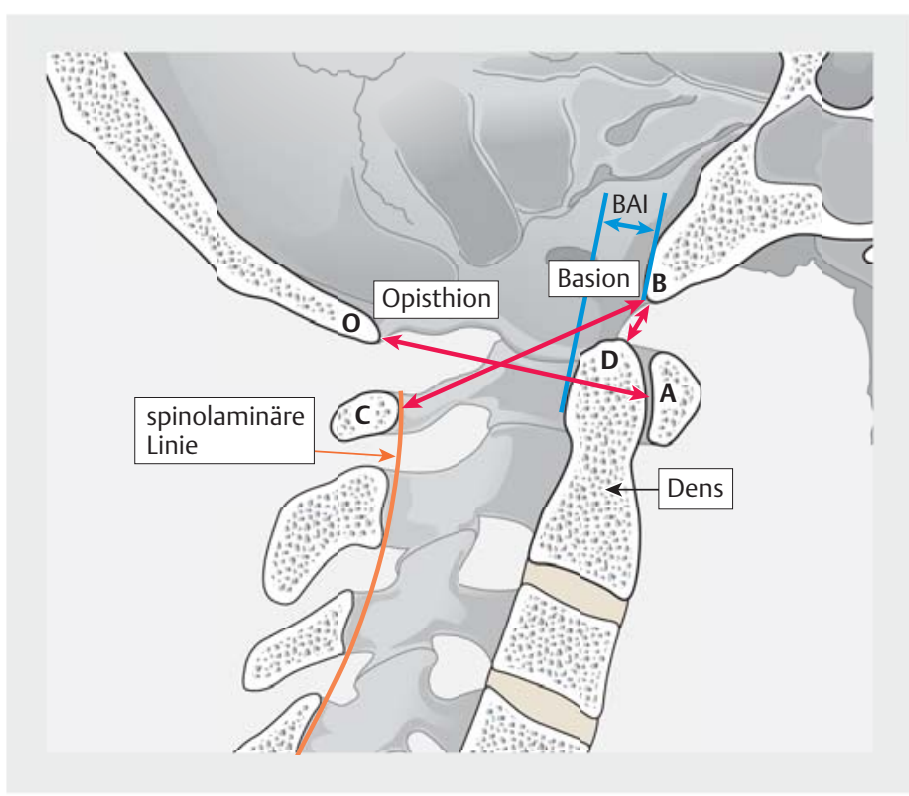

Abb. 16 Einige wichtige Hilfslinien und Maße an der okzipitozervikalen Junktion (vgl. Harris, 1994 [74, 75]). AO/BC (sog. Power's Ratio [95]) sollte stets < 1 sein (normal: 0,77); das Dens-Basion-Intervall (BD) nicht $>12$ mm (über 13 Jahre); BAl: Basion-Axis-Intervall: zw. +12 und -4 mm. 

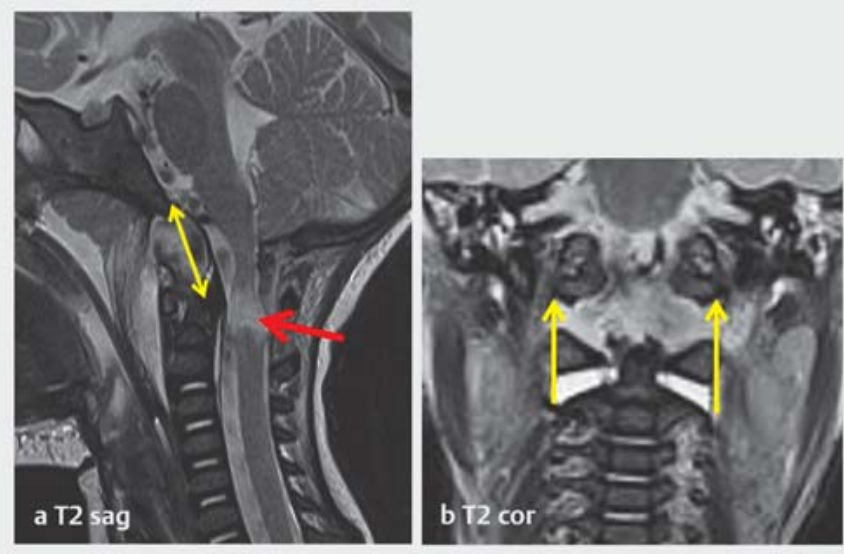

- Abb.17 Okzipitozervikale Separation: 3-jähriges Kind, Autounfall: a Vollständige kraniozervikale Disruption aller Bänder (Doppelpfeil) bis auf Membrana tectoria mit Transsektion des oberen Halsmarkes (roter Pfeil); b Zusätzlich auch atlantoaxiale Disruption bds. (Pfeile).

tur; $\vee$ Abb. 19 a,b). Geschieht dies an 2 oder mehreren Lokalisationen des Atlasringes gleichzeitig und vollständig, d.h. mit Quertranslation der Fragmente $(>7 \mathrm{~mm}$, „Rule of Spence“; [89]), so entsteht infolge obligater Zer- reißung des Lig. transversum atlantis eine instabile Situation (klassische Jefferson-Fraktur; [35]; > Abb. 20). Sie entspricht dem Typ III in der Einteilung nach Gehweiler et al. [90], während alle anderen atlantalen Frakturen als stabil gelten, sofern mögliche assoziierte Frakturen (vor allem des Axis) diese Einschätzung zulassen. Landells et al. fügten 1988 eine eigene Klassifikation hinzu [91], die auf die Bedeutung der Integrität des Lig. transversum atlantis besonderen Bezug nimmt. Hier kann auf den wichtigen Stellenwert des intakten Lig. transversum bei der Atlasintegrität und -stabilität nicht weiter eingegangen werden; eine entsprechende Einteilung gaben u. a. Dickman et al. 1996 [86]. Neurologische Defizite sind bei isolierten Atlasfrakturen nicht oder nur selten anzutreffen, jedoch ist die sog. Atlasschlinge (Gefäßschlinge der Vertebralarterie[n]) in Gefahr, traumatisch geschädigt zu werden (Dissektion, Thrombose) [36].

\section{Densfrakturen}

Sie stellen die häufigsten spezifischen Frakturen der oberen HWS dar [37]. Grund hierfür ist einerseits seine einzigartige anatomische Konfiguration und Lage, welche den Dens gegenüber frakturverursachenden Mechanismen exponiert und anfällig macht ( $\bullet$ Abb. 21) [38]. Andererseits prädestiniert die relative knöcherne Rarefizierung im Basisbereich des Dens axis dazu, dass sich die meisten Frakturen an dieser "Sollbruchstelle“ des Dens
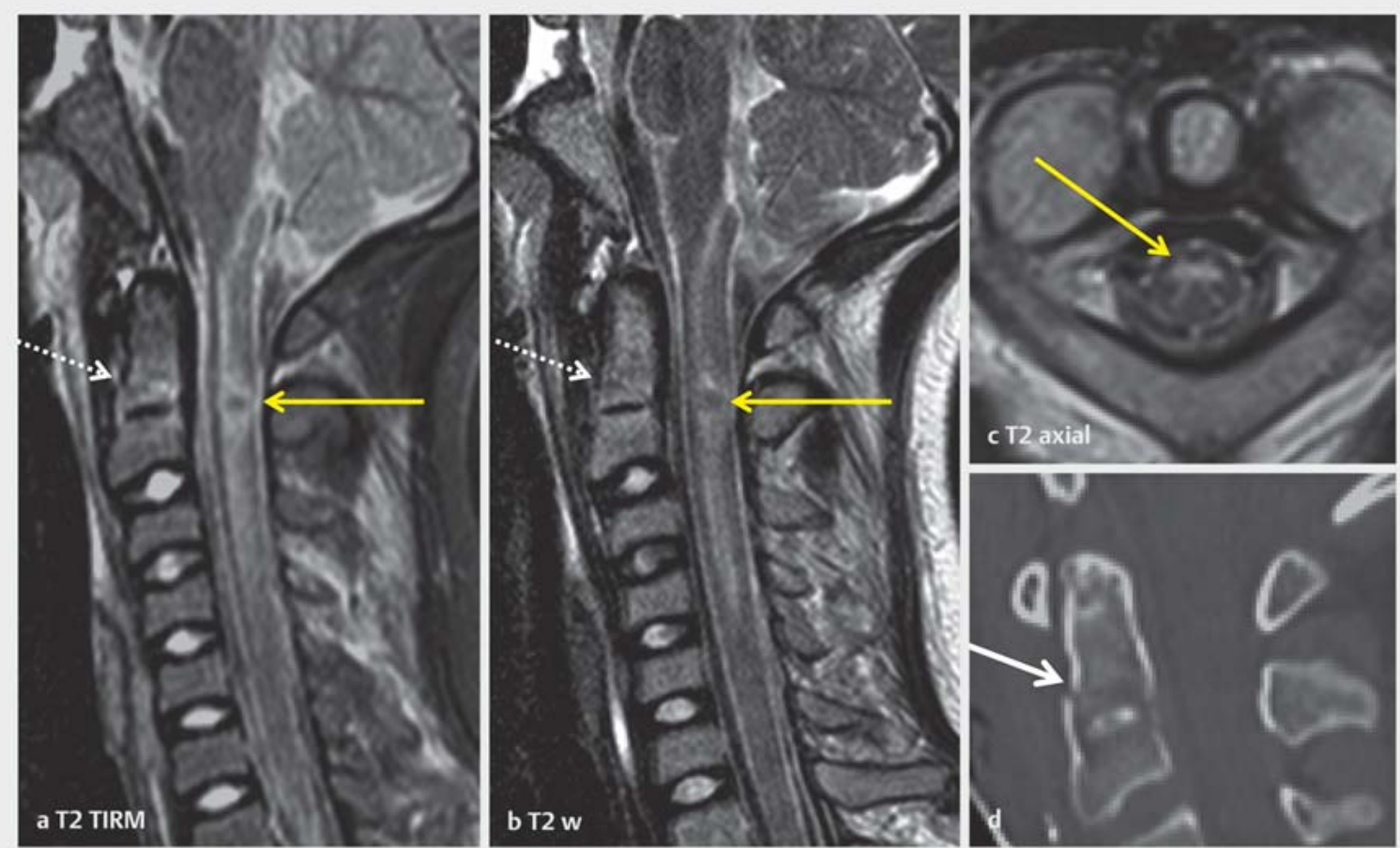

- Abb. 18 SCIWORA: 14-jähriger Junge, Verkehrsunfall (Pkw-Beifahrer), Tetraparese. Es zeigt sich eine hochzervikale Myelopathie (gelber Pfeil in c), möglicherweise mit einer medullären Hämorrhagie (gelbe Pfeile in a + b). CAVE: das initial erfolgte HWS-Röntgen war unauffällig (nicht gezeigt); erst das nach der MRT durchgeführte CT ergab tatsächlich eine Densfraktur! (weißer Pfeil in d), die retrospektiv auf dem MRT bereits zu vermuten war (gestrichelte Pfeile, $\mathbf{a}+\mathbf{b}$ ). 

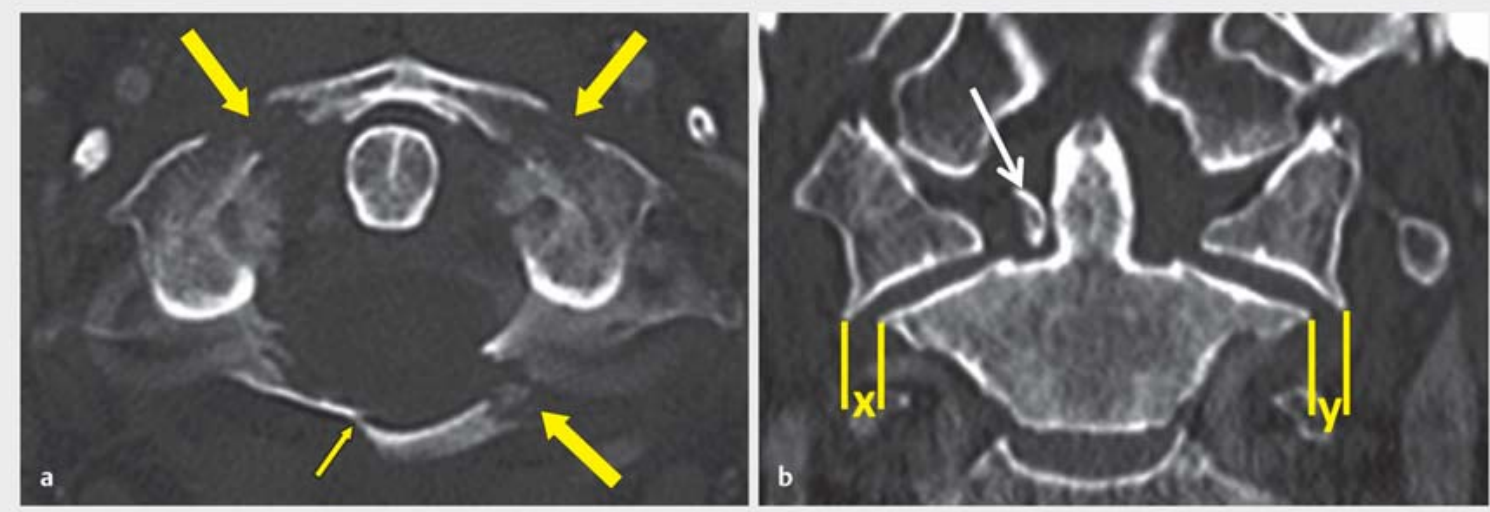

- Abb. 19 a Klassische Jefferson-Fraktur: sog. 4-Part-Fracture (Pfeile) mit Frakturspaltdistraktionen. b Klassische Jefferson-Fraktur, identischer Patient: Malalignment der Massae laterales atlantis ( $x+y>7$ mm: „Rule of Spence“): knöcherner Ausriss des Lig. transversum atlantis (weißer Pfeil) $\rightarrow$ instabile Fraktur! Quelle: Grieser T, Fessl R. Wirbelsäule. In: Bohndorf K, Imhof H, Wörtler K. Radiologische Diagnostik der Knochen und Gelenke. 4. Aufl. Stuttgart: Thieme; 2017: 79-105.
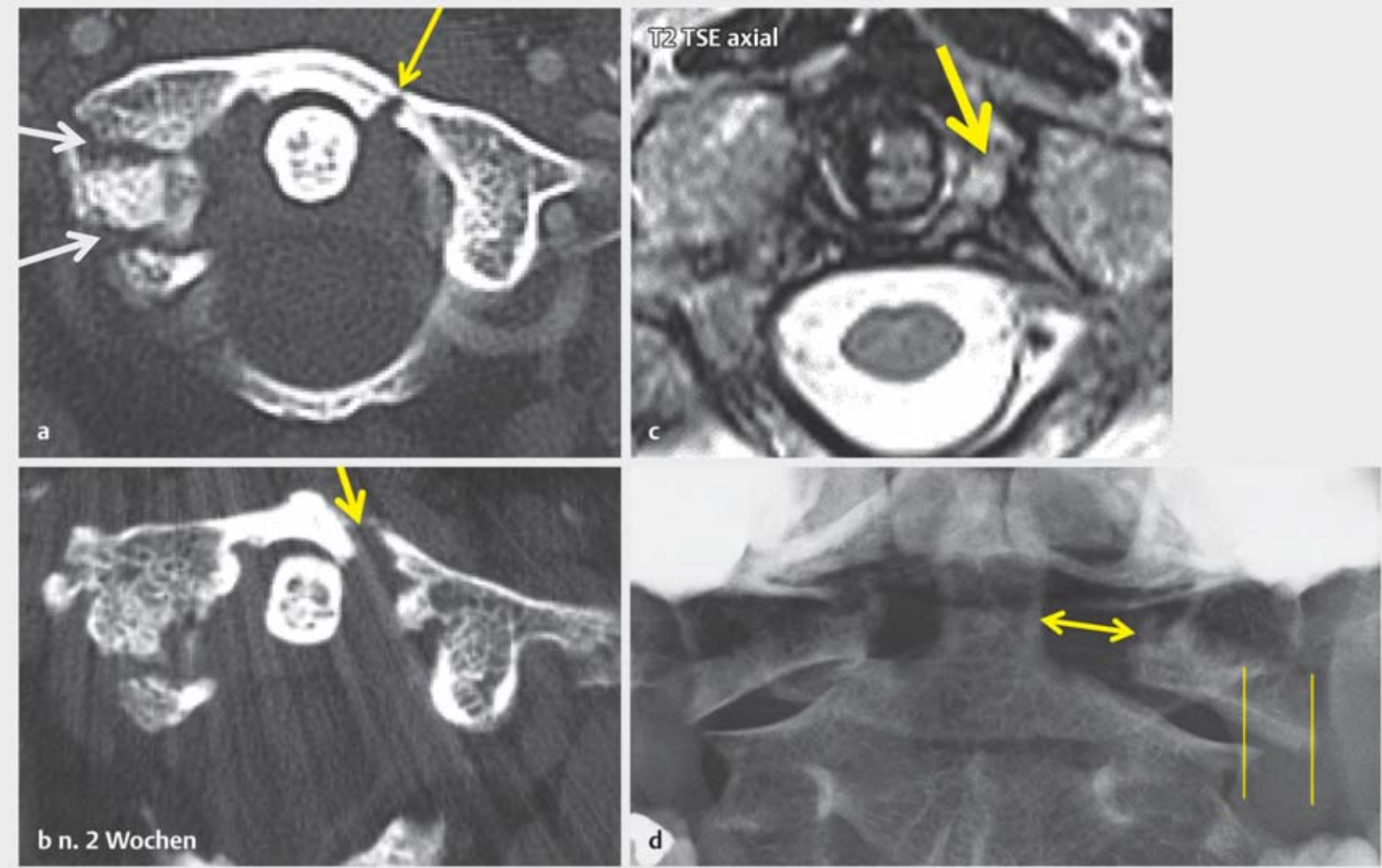

- Abb. 20 Bilaterale Atlasfraktur (Pfeile in a), die im Verlauf eine zunehmende Dehiszenz erfahren hat (Pfeil in b); die MRT zeigt eine linksseitige Avulsion des Lig. transversum atlantis (gelber Pfeil in c); $\mathbf{d}$ „Atlasverbreiterung“: Rule of Spence (unilateral; Doppelpfeil, Linienpaar).

ereignen (instabiler Typ II in der Einteilung nach Anderson und D’Alonzo von 1974; [39]; > Abb. 22). Dennoch bleibt die Densfraktur aufgrund zahlreicher Eigenheiten „rätselhaft" und Gegenstand weiterer Forschung und Beobachtung [40]. Myelonschäden sind aufgrund des relativ großen Sagittaldurchmessers auf Höhe des Foramen magnum zwar primär selten, können aber aufgrund sekundärer Dislokationen und Non-Union-bedingter Instabilitäten zur Kompressionsmyelopathie führen. Aufgrund der Tatsache, dass praktisch nur die Typ-2-Densfrakturen 

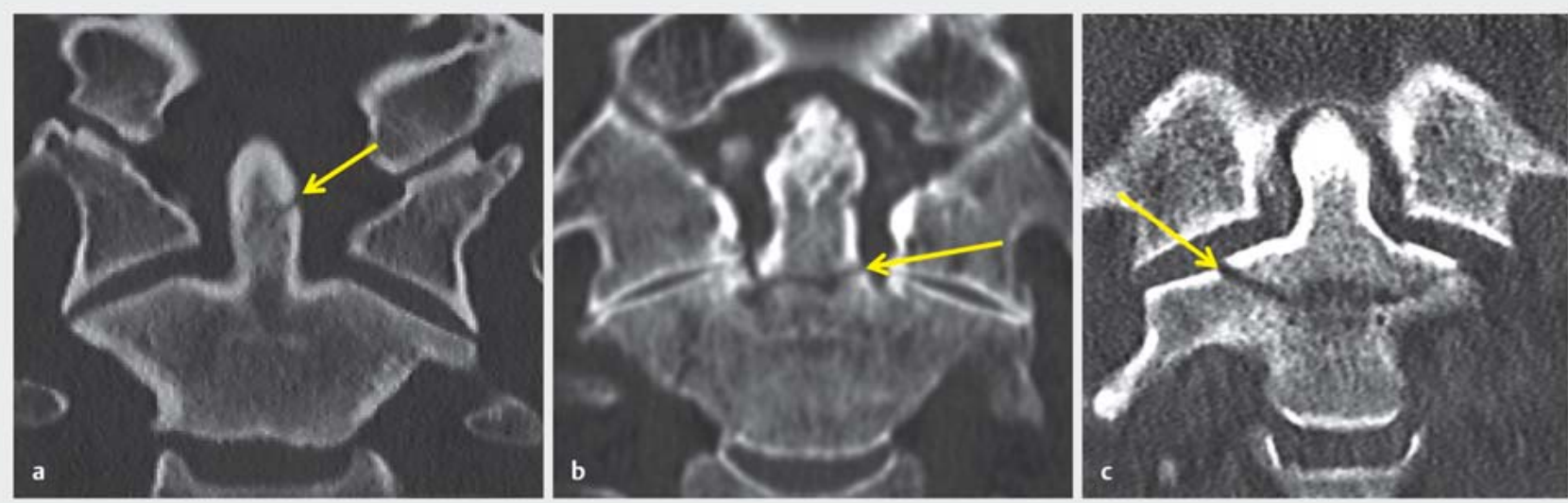

- Abb. 21 Drei Beispiele für undislozierte Densfrakturen: a Typ I: Schrägfraktur durch die Densspitze. b Typ II: Querfraktur durch die Densbasis. c Typ III: bogenförmige Fraktur durch den Corpus axis.
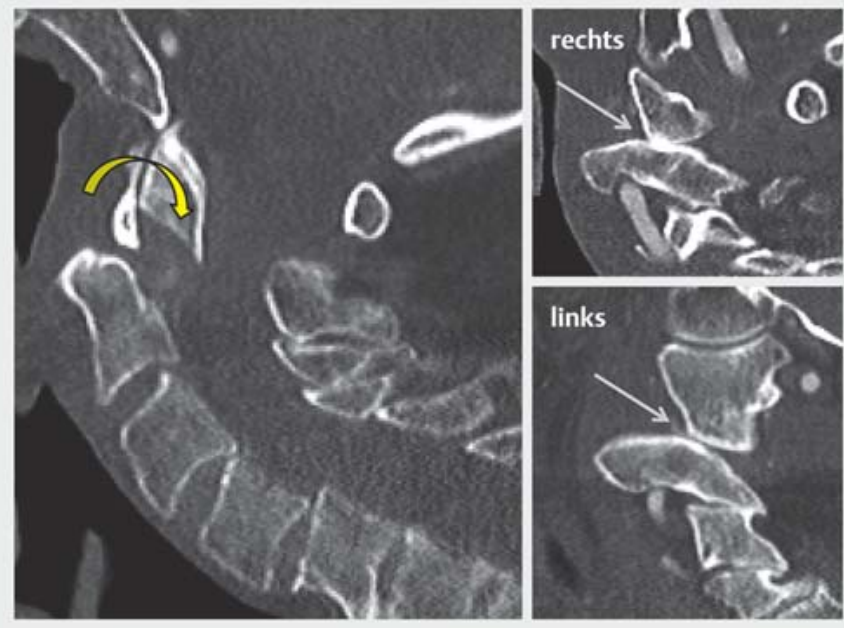

- Abb. 22 Dislozierte Densfraktur bei einem 67-jährigen Mann (Sturz): Densfraktur Typ II nach Anderson und D'Alonzo (Subtyp 2 nach Roy-Camille) mit starker Dorsaldislokation und bilateraler atlantoaxialer Luxation.

zu relevanten Komplikationen (Dislokation, Pseudarthrose) führen, unterteilten Roy-Camille et al. 1980 diesen Frakturtyp nochmals hinsichtlich seines Frakturverlaufes, um eine Vorhersage der Dislokationsrichtung zu treffen [92]. Dabei stellen nach dorsal angulierte oder horizontal verlaufende Frakturverläufe einen Prädiktor für instabile und nach dorsal dislozierende Densbrüche mit konsekutiver Myelongefährdung dar.

Ein weit größeres Problem stellen resultierende atlantodentale Instabilitäten dar, die zumeist Folge nicht verheilter Densfrakturen (instabile Denspseudarthrosen) sind. Zu ihrer Diagnose werden sog. Funktionsaufnah- men (Flexions-/Hyperextensionsaufnahmen) angefertigt, die auch in der MRT (zumindest in Flexionsstellung) durchgeführt werden können. Differenzialdiagnostisch sind Denspseudarthrosen gegen (instabile) Nearthrosen eines großen Os odontoideum abzugrenzen [41]. Auf entzündlich bedingte atlantodentale Instabilitäten, wie sie insbesondere bei der rheumatoiden Arthritis beobachtet werden können, sei hier nur hingewiesen [42,43].

\section{Hangman's Fracture}

Einen weiteren nur an der oberen Halswirbelsäule anzutreffenden Frakturtyp stellt die sog. Hangman's Fracture dar. Auf den Ursprung dieser geläufig gewordenen Bezeichnung, die eigentlich als traumatische Spondylolisthese des Axis bezeichnet wird, kann hier nicht näher eingegangen werden, wichtig ist aber, dass diese Fraktur den isthmischen Teil des Atlasbogens, die Pars interarticularis axis, betrifft ( $\triangleright$ Abb. 23 ). Während die traumatische Spondylolisthese allein nur ca. 5\% aller zervikalen Verletzungen ausmacht, soll sie in immerhin 30\% aller zervikalen Frakturen begleitend beobachtet werden [44], was dem Autor ungewöhnlich hoch erscheint. Stark hyperextendierende Traumamechanismen zusammen mit einer distrahierenden Komponente führen zu einer bilateralen, dabei aber oft asymmetrischen Fraktur der Bögen bzw. Bogenwurzeln des Axis; mitunter verläuft die Fraktur teilweise asymmetrisch durch Teile des Axiswirbelkörpers, wodurch atypische Varianten zur klassischen Einteilung nach Effendi et al. [93] bzw. Levine und Edwards [94]) entstehen. Jedoch ist weniger die bilaterale Bogenfraktur das Problem dieser Verletzung, sondern die mögliche Mitbeteiligung der subaxialen Bandscheibe bei C II/III [45]. Ist diese mitbetroffen, d. h. relevant rupturiert, so resultiert eine segmentale Instabilität, die je nach ihrem radiologischen Ausmaß unterschiedlich graduiert wird. Aus diesem Grunde sollte der diskalen bzw. 

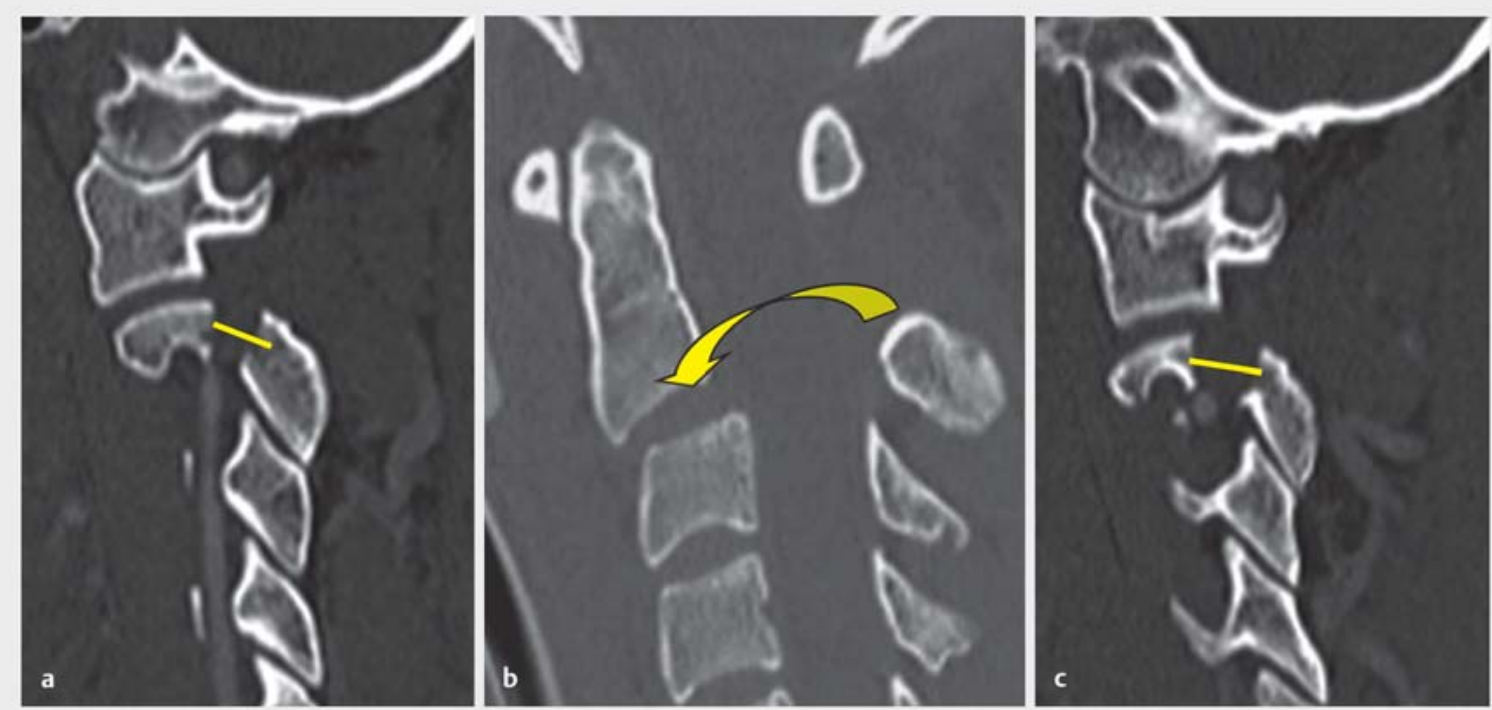

- Abb. 23 Klassische Hangman's Fracture: traumatische bilaterale Spondylolisthesis (gelbe Strichmarkierungen in a und c) mit ventraler Angulation des Dens einschl. Denskorpus (gebogener Pfeil in b): Typ II a n. Levine/Edwards. Quelle: Grieser T, Fessl R. Wirbelsäule. In: Bohndorf K, Imhof H, Wörtler K. Radiologische Diagnostik der Knochen und Gelenke. 4. Aufl. Stuttgart: Thieme; 2017: 79-105
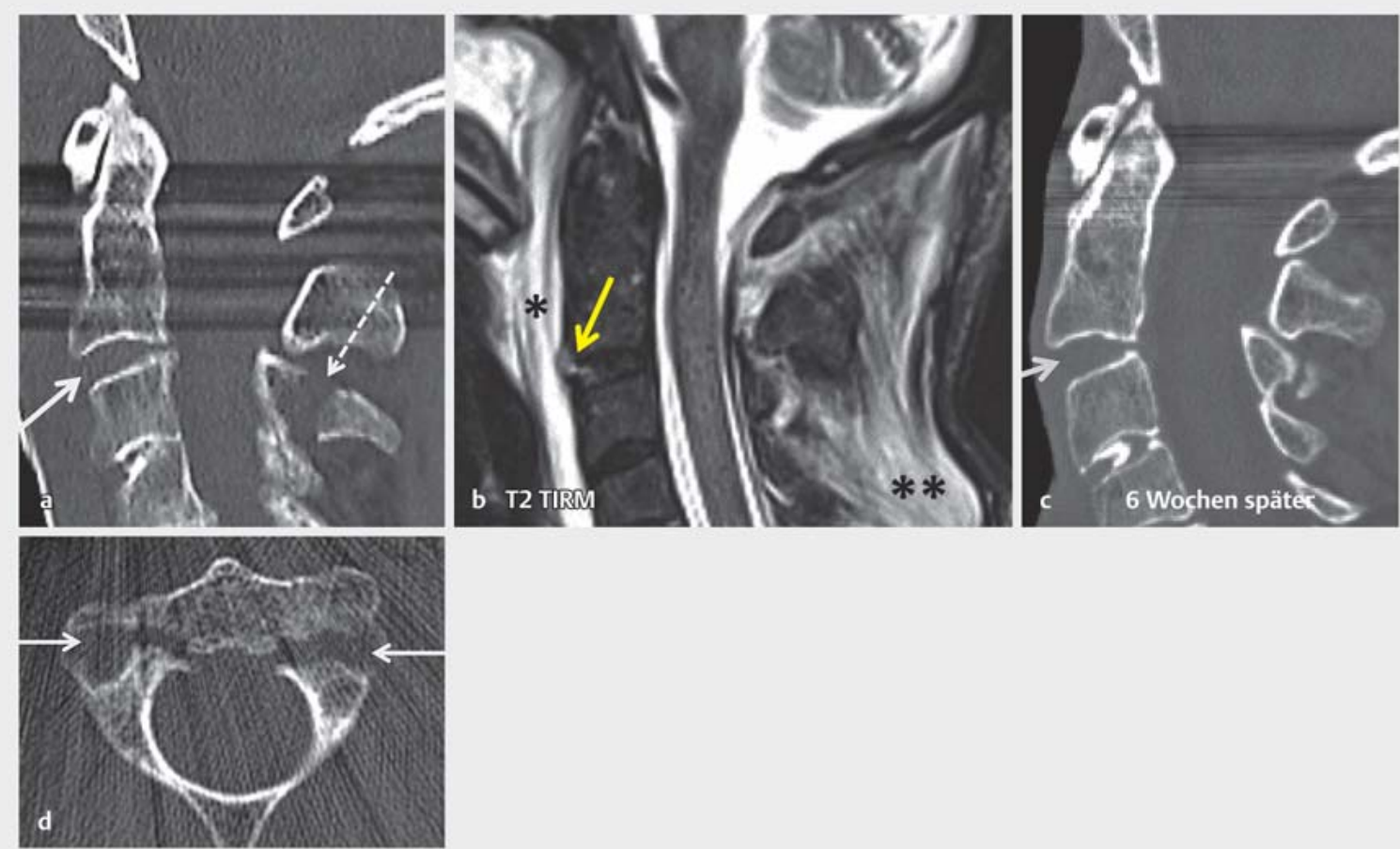

- Abb. 24 Hangman's Fracture von C II: Fraktur durch die korpusnahen Bogenwurzeln (Pfeile in d); Abbruch des Dornfortsatzes von C III (gestrichelter Pfeil in a; Hyperextensionsmechanismus). a Im CT ventrales Klaffen des C-II/III-Bandscheibenfaches (Pfeil); in der MRT Zerreißung des Anulus und des vorderen Längsbandes (gelber Pfeil in b). Zusätzlich deutliches prävertebrales und nuchales Ödem $\left({ }^{*},{ }^{* *}\right)$. c 6 Wochen später leichte Zunahme der ventralen C-II/III-Hyperextension (kurzer Pfeil). 

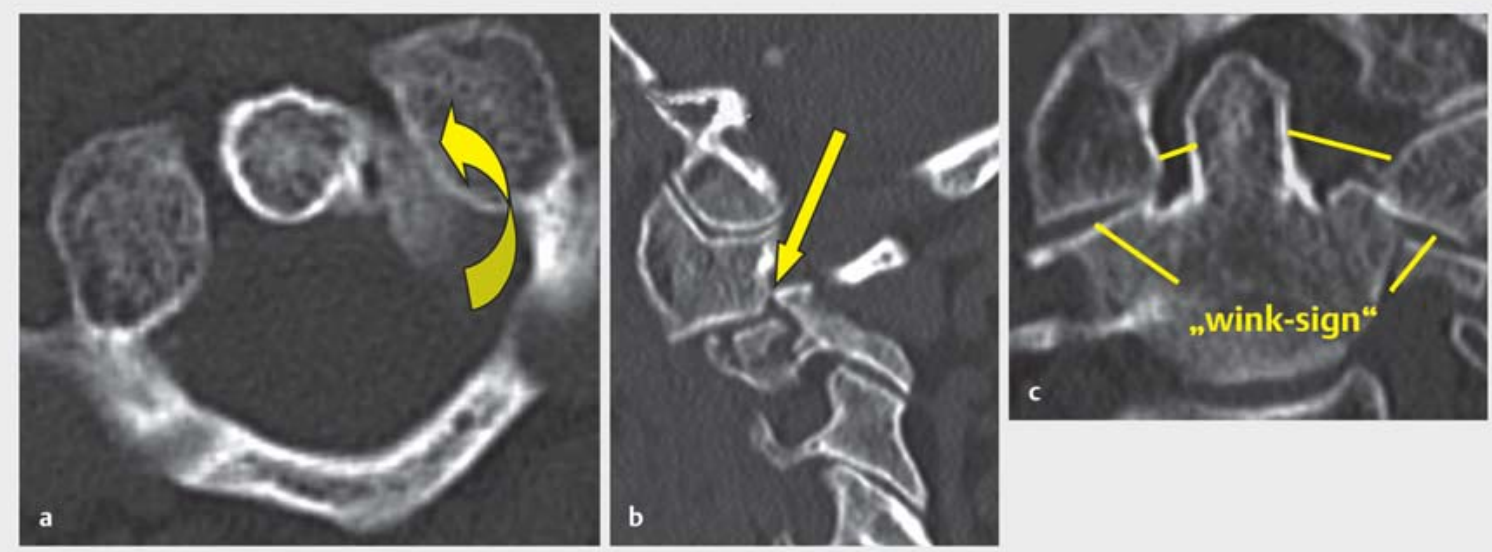

- Abb. 25 Beispiel einer rein rotatorischen atlantoaxialen (einseitigen) Fehlstellung. a asymmetrische Rotationsfehlstellung links (gebog. Pfeil). b Ursache ist eine infolge Gelenkfacettenfraktur „verhakte“ Massa lateralis atlantis (Pfeil). c Sowohl röntgenologisch als auch im CT auffallende Gelenkspaltasymmetrie („BlinzelZeichen“) sowie asymm. atlantodentale Distanzen (gelbe Striche). Quelle: Grieser T, Fessl R. Wirbelsäule. In: Bohndorf K, Imhof H, Wörtler K. Radiologische Diagnostik der Knochen und Gelenke. 4. Aufl. Stuttgart: Thieme; 2017: 79-105.

diskoligamentären Integrität mittels MRT große Bedeutung beigemessen werden ( $\triangleright$ Abb. 24) [46].

\section{Atlantoaxiale rotatorische Verletzungen}

Eine besonders große Herausforderung stellt die bildgebende Diagnostik atlantoaxialer rotatorischer Verletzungen dar, weil sich bereits physiologische Bewegungsausschläge mit bis zu $40^{\circ}$ mit pathologischen Rotationsfehlstellungen dieser Größenordnung überlagern können und eine Differenzierung somit schwerfällt [47]. Zwar sind echte und rein rotatorische Subluxationsstellungen meistens fixiert und im Unterschied zu willkürlichen Kopfdrehstellungen nicht ohne Weiteres beheb- bzw. korrigierbar (Abb. [25]), eine klarere Aussage bez. echter Rotationsfehlstellungen kann aber stets dann gegeben werden, wenn zusätzlich eine pathologische anteriore Atlasdislokation besteht (Typen 2-4 nach Fielding und Hawkins; [48]).

\section{Atlantoaxiale bzw. atlantodentale sagittale Instabilitäten}

Eine weitere Besonderheit der oberen HWS stellen die atlantoaxialen bzw. atlantodentalen sagittalen Instabilitäten dar (Klassifikation n. Wang et al., 2013; [76]). Auf die instabile Denspseudarthrose wurde weiter oben schon hingewiesen, zudem gibt es aber auch atlantoaxiale Dislokationen als posttraumatische Folgezustände, bei entzündlicher Affektion (z. B. rheumatoider Arthritis, Kristallarthropathien; vgl. - Abb. 3) oder bei anlagebedingter kraniozervikaler Übergangsstörung (großes Os odontoideum, okzipitodentale Fusionsanomalie etc.; $>$ Abb. 26). Diese Veränderungen sind dann bedeutsam, wenn sie über einen Kneifzangenmechanismus zu einer zunehmenden hochzervikalen Spinalkanalstenose mit konseku- tiver Myelonkompression führen. Dies erfordert dann i.d.R. eine chirurgische kraniozervikale Dekompression mit obligater (dorsaler) Stabilisierung.

Auf die Besonderheiten der normalen und der verletzten kindlichen HWS kann hier im Rahmen dieses Beitrages nicht weiter eingegangen werden.

\section{Die subaxiale HWS (C III-C VII)}

Neben den oben besprochenen, besonderen Frakturtypen und -mechanismen der oberen HWS stellt die subaxiale HWS aufgrund ihrer freien Beweglichkeit einen besonders traumaexponierten Teil des gesamten Achsenskeletts dar, wobei hier die untere HWS (C V-C VII) sowie der zervikothorakale Übergang zahlenmäßig am häufigsten einer traumatischen Schädigung unterliegen [49]. Dies wird mit dem mechanischen „Peitschenschlagprinzip“ erklärt, wobei die obere BWS aufgrund der kostalen Fesselung und Stabilisierung das fixe Widerlager gegen die stark ausschwingende HWS bildet, was die besondere Belastung der Segmente HWK V-VII erklärt: Immerhin betreffen über die Hälfte aller zervikalen Verletzungen die Segmente HWK V/VI und HWK VI/VII [49].

Grundsätzlich unterliegen die Verletzungsmuster der subaxialen HWS den gleichen Prinzipien wie die der thorakalen und lumbalen Wirbelsäule, jedoch gibt es - sinnvollerweise - hierfür ein eigenes Klassifikationssystem (AO Subaxial Cervical Spine Injury Classification System; [50]). Dieses hebt, neben den bekannten Frakturmorphologietypen (A-C; Kompression, Spaltbruch, Berstung; Flexion- u. Hyperextension-Distraktion; Rotation), insbesondere auf die Beurteilung von diskoligamentären Läsionen $a b$, womit wiederum der MRT als entscheidender 

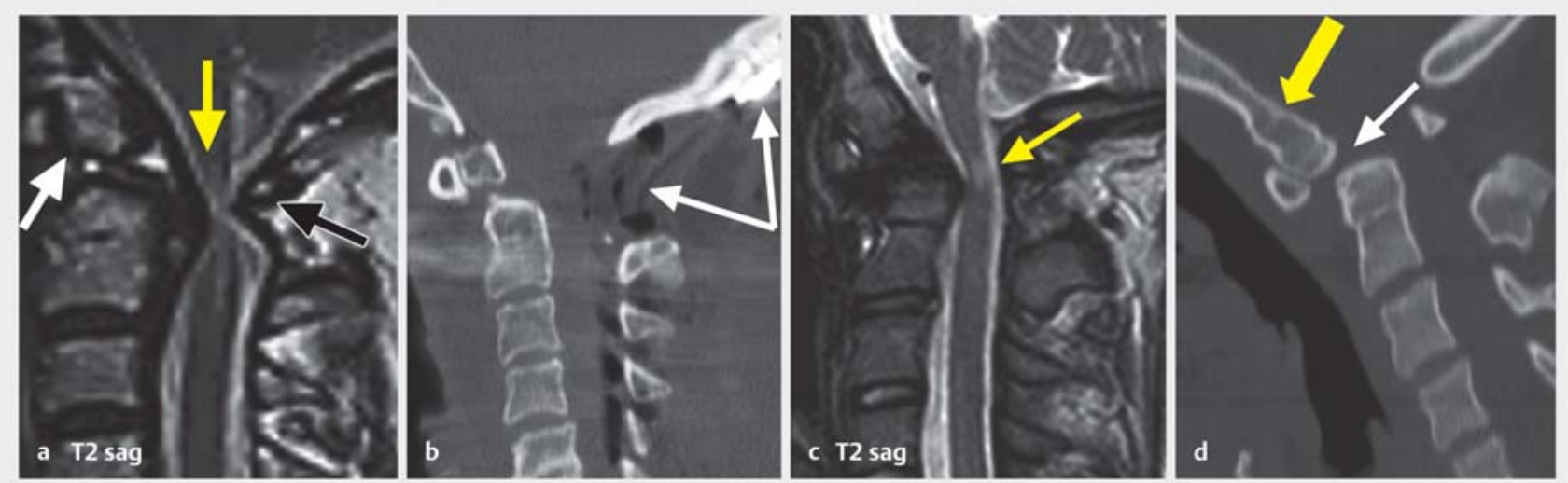

- Abb. 26 Instabile Denspseudarthrose (DD Os odontoideum): 68-jährige Patientin mit fortschreitender, zuletzt hochgradiger Tetraparese: a Hochgradige Spinalkanalstenose auf Höhe C I mit Myelopathie (gelber Pfeil) mit Einschnürung des Spinalkanals durch den hinteren Atlasring (schwarzer Pfeil); nach ventral angulierte Denspseudarthrose oder Os odontoideum (weißer Pfeil). b Postoperative Situation nach okzipitozervikaler Fusion und Bogenresektion C I (Pfeile); c + d) kraniozervikale Übergangsanomalie: 15-jähriges Mädchen mit Dys- und Parästhesien sowie Bewegungsschmerz: c Myelopathiesignal im oberen Halsmark (Pfeil). d Ursache ist ein mit dem Basion verschmolzenes Os odontoideum (gelber Pfeil), was zu einer Überlastung = Hypermobilität in der Nearthrose führt (weißer Pfeil).

diagnostischer Modalität ein höherer Stellenwert zukommt als an den übrigen WS-Abschnitten. Des Weiteren gehen auch Fehlstellungen und Frakturen der Facettengelenke mit in die Beurteilung ein, was aufgrund der großen zervikalen Beweglichkeit durchaus von Bedeutung ist (sog. F-Grading). Die klassifikationsgemäße Beurteilung des neurologischen Status ( $\mathrm{NO}-\mathrm{Nx}$ ) ist zwar i. d. R. von radiologischer Seite nicht zu leisten, viel wichtiger indes erscheinen aber die neu eingeführten patientenspezifischen Variablen (sog. Modifier, M1-M4), die neben diskoligamentären Läsionen und Verletzungen der A. vertebralis vor allem das Vorliegen einer versteifenden Wirbelsäulenerkrankung (entzündlich oder degenerativ) einschließt. Einen umfassenden Überblick über diese hier nur kurz skizzierten Frakturmechanismen und ihre Klassifikation findet sich in Band 5 der AO Spine Masters Series von 2015 [72].

Direkt abgeleitet aus diesem o. g. neuen, im wesentlichen morphologisch basierten AO-C-Spine-Klassifikation wurde der SLIC-Verletzungsschwere-Score (SLIC: Subaxial Injury Classification) [80]. Dieser Score (berücksichtigt neben den radiologischen Kriterien der knöchernen vertebralen Pathomorphologie und der diskoligamentären Schädigung auch den neurologischen Status) soll dabei im wesentlichen eine Entscheidungshilfe für Chirurgen sein, bei welcher Verletzungsschwere eine operative Intervention angezeigt ist. Obwohl sicher nicht in jedem Szenario perfekt, zeigte das SLIC-System eine exzellente Intra- und Interobserver-Übereinstimmung (>90\%) und erwies sich als sicherer und effektiver Behandlungs-Guide hinsichtlich des Erhalts des neurologischen Status des Patienten $[81,82]$.
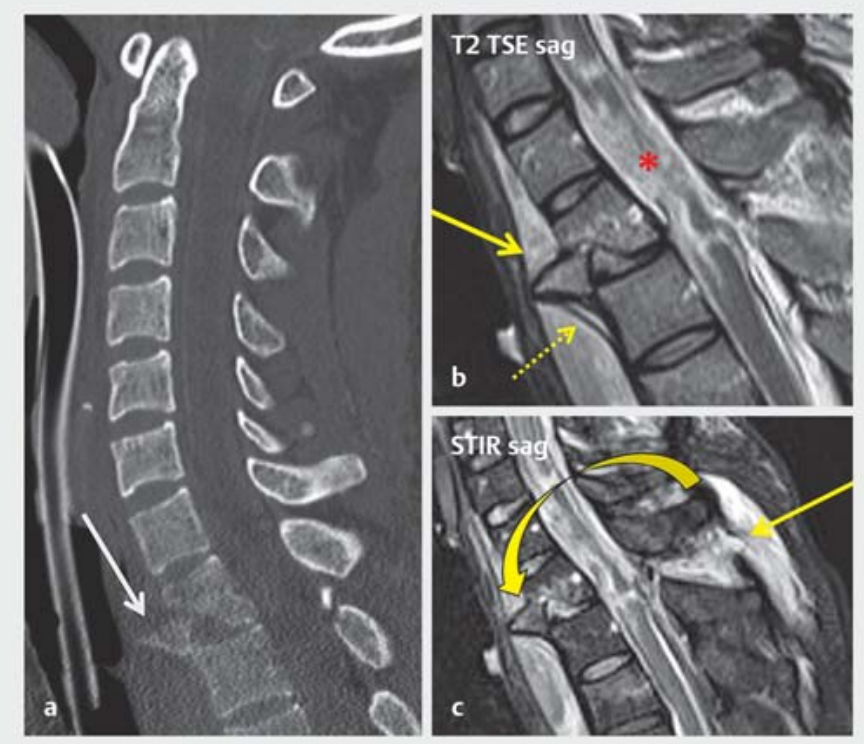

- Abb. 27 Ausgeprägter Flexions-Teardrop bei BWK I: dislozierte Aussprengung der Vorderunterkante von BWK I (Pfeil in a); in der MRT-Darstellung des rotierten Teardrop-Fragmentes mit Ruptur des vorderen Längsbandes (Pfeile in b) sowie Zerreißung des unteren Halsmarkes $\left({ }^{*}\right)$; die STIR-Sequenz zeigt zusätzlich die Flexionskomponente (gebogener Pfeil) mit Zerreißung der dorsalen ligamentären Zuggurtung (Pfeil in c).

Einige typische zervikale Verletzungsmuster

\section{Teardrop-Frakturen}

Hierbei unterscheidet man wiederum die Flexions-Teardrop-Frakturen von den Hyperextensions-Teardrop-Frakturen. Erstere entstehen, wenn neben einer stark flektie- 


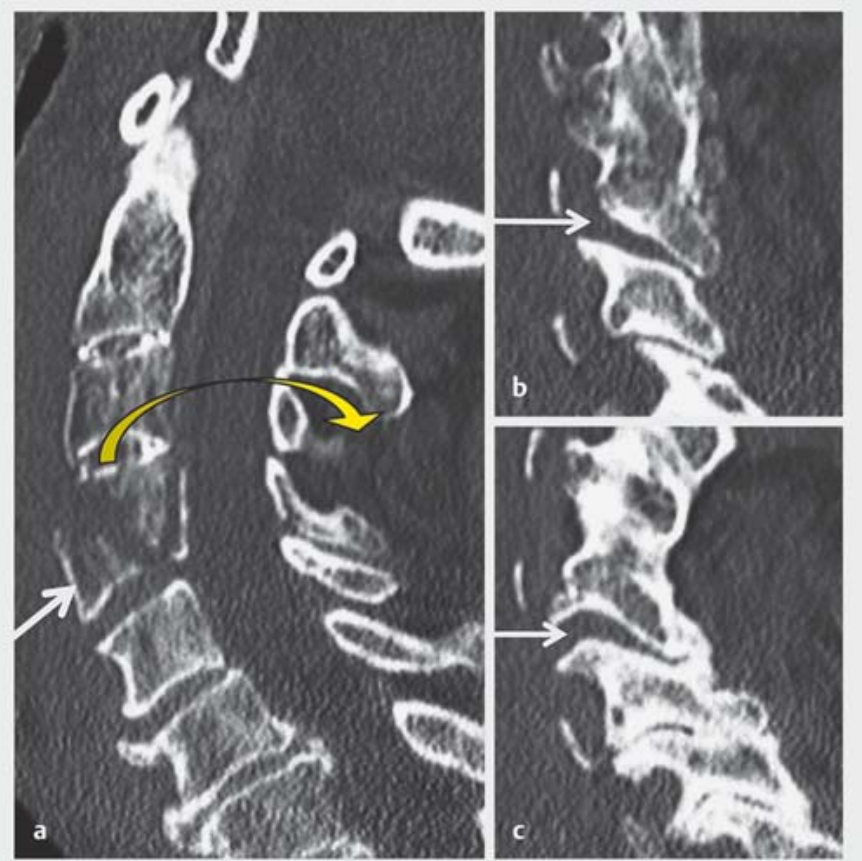

- Abb. 28 Hyperextensions-Teardrop-Fraktur (gebogener Pfeil) bei teilankylosierter Wirbelsäule mit großem Teardrop-Fragment (Pfeil in a); zusätzlich bds. klaffende Facettengelenke (Pfeile in $\mathbf{b}$ und $\mathbf{c}$ ). renden Kraft zeitgleich auch eine axiale Stauchung wirkt, sodass es im typischen Falle zu einer Absprengung eines mehr oder weniger großen Vorderunterkantenfragmentes kommt ( $\triangleright$ Abb. 27). Gleichzeitig erfolgt eine Dorsaldislokation des posterioren WK-Fragmentes in Richtung Spinalkanal, während sowohl Bandscheibe, posteriores Längsband und die dorsale Zuggurtung zerrissen werden können; es resultiert eine hochgradig instabile Verletzungssituation [51]. Hyperextensions-Teardrop-Frakturen stellen Avulsionsfragmente der WK-Vorderunterkante, typischerweise bei HWK II, dar, die durchaus schalenartig schmal sein können, aufgrund ihres Hyperextensionsmechanismus aber schwere diskoligamentäre Distraktionsschäden signalisieren. Indirekte Röntgenbzw. CT-Zeichen können die erweiterten anterioren intervertebralen Abstände, Facettengelenks- oder Dornfortsatzfrakturen sein ( $\triangleright$ Abb. 28). In jedem Fall resultieren auch hier spinale Instabilitätszustände [52]. Wegen der subtilen radiologischen Frakturzeichen ist umso mehr auf die prävertebrale Weichteilschwellung sowie auf eine mögliche traumatische Retrolisthese zu achten. Hierzu machten Korres et al. 2007 einen Klassifikationsvorschlag (Typen I-IV), wobei insbesondere auf das Ausmaß der dorsalen Fragmentdislokation Wert gelegt wurde [53].

\section{Sub-/Luxationen}

Die eingangs schon erwähnte hohe Mobilität der HWS in allen 3 Freiheitsgraden hat auch eine starke Subluxations-/Luxationsneigung zur Folge ( $\mathbf{A b b . 2 9 )}$ ), deren

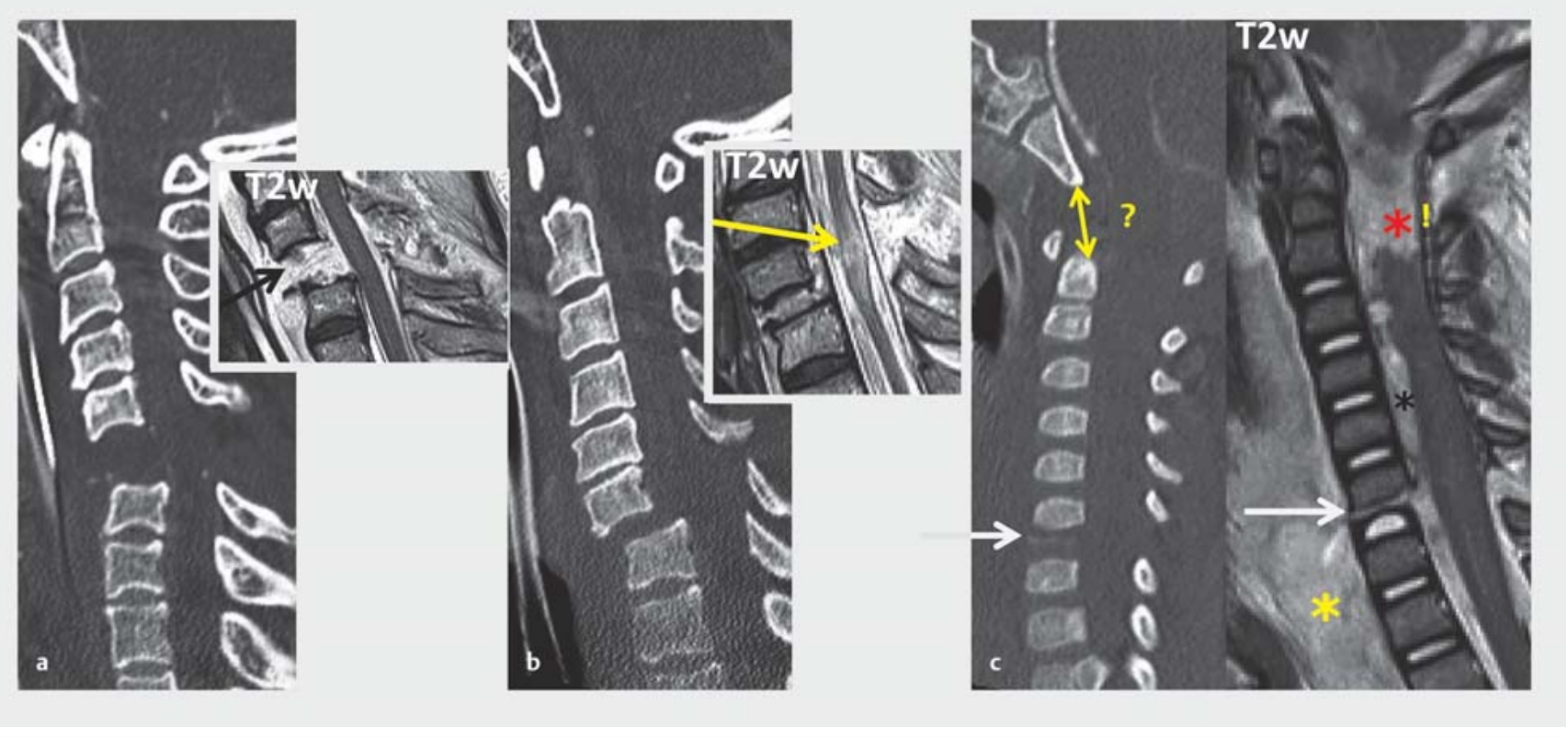

- Abb. 29 Drei Fallbeispiele subaxialer Luxations-Distraktions- bzw. -Flexions-Frakturen: a 59-jährige Frau mit vollständiger diskoligamentärer Zerreißung (Pfeil), Myelon jedoch morpholog. intakt. b 48-jähriger Mann mit asymm. Luxationsfraktur und schwerer Myelonkontusion (Pfeil). c Zweijähriges Kind: auffälliges Spacing bei C VI/VII (Pfeil) sowie fraglich abnormal weites Dens-Basion-Intervall (Doppelpfeil): die MRT zeigt neben einer physealen Zerreißung der Grundplatte C VI (Pfeil) auch eine vollständige Myelontranssektion (roter *). Beachte das prävertebrale (gelber *) und epidurale Hämatom (schwarzer *). Das Kleinkind verstarb 3 Tage post eventum. 
Wahrnehmung durch die mitunter schon wieder eingetretene Reposition zum Untersuchungszeitpunkt erschwert wird. Wird diese „Spontanreposition“ durch Verhakung der luxierten Facettengelenke verhindert, so wird diese Gelenkinkongruenz auf sagittalen Rekonstruktionen ohne Weiteres erkennbar. Dabei ist die beidseitige Facettengelenksluxation hinsichtlich ihrer segmentalen Stabilität kritischer einzuschätzen als die einseitige [54]: Letztere wird auch als sog. „interlocking“ bezeichnet, d. h. sie ist aufgrund ihrer diagonalen Verblockung in einseitiger Fehlstellung wiederum relativ stabil ( $\mathbf{A}$ bb. $\mathbf{3 0}$ ). Allerdings liegt diesem Verletzungsmuster eine rotatorische Komponente zugrunde, der wiederum ein höheres Instabilitätspotenzial innewohnt [57].

Jede offensichtliche monosegmentale intervertebrale Distanzierung, horizontale Translation (>3,5 mm) oder vermehrte Angulation ( $>11^{\circ}$ in sagittaler Ebene) haben bis zum Beweis des Gegenteils als Zeichen der segmentalen Instabilität zu gelten [55], wenngleich insbesondere bei älteren Patienten degenerative Veränderungen derartige suspekte Stellungsänderungen vortäuschen können (Pseudospondylolisthese), ebenso gelten die schon fast historischen, bildhaften Facettengelenksluxationszeichen auf axialen CT-Schichten (reverse Hamburger Bun Sign, Naked Facet Sign bzw. Headphone Sign; [56]; s. - Abb. 28) als sichere Luxationszeichen.

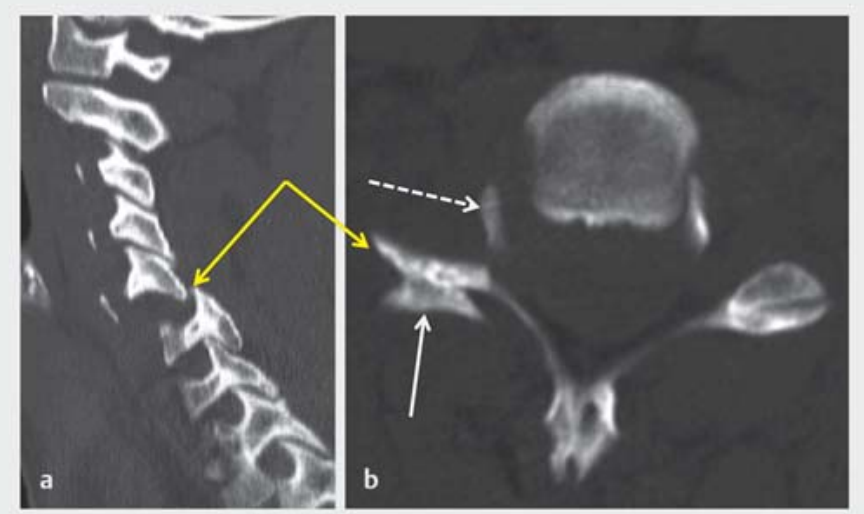

Abb. 30 Interlocking: 46-jährige Frau, Verkehrsunfall, Parästhesien beider Arme/Hände: a Einseitige Facettengelenksluxation rechts (Pfeil). b Ipsilaterale Facettenluxation rechts (reverse Hamburger Bun Sign; weiBer Pfeil) sowie zusätzlich asymmetrische Unkovertebralgelenke (einseitiges Headphone Sign; gestrich. Pfeil). Quelle: Grieser T, Fessl R. Wirbelsäule. In: Bohndorf K, Imhof H, Wörtler K. Radiologische Diagnostik der Knochen und Gelenke. 4. Aufl. Stuttgart: Thieme; 2017: 79-105.

Von viel wichtigerer praktischer Bedeutung ist die Beachtung asymmetrischer, d. h. einseitiger Frakturen der Pedikel, Facettengelenke, Querfortsätze und auch der Rip-
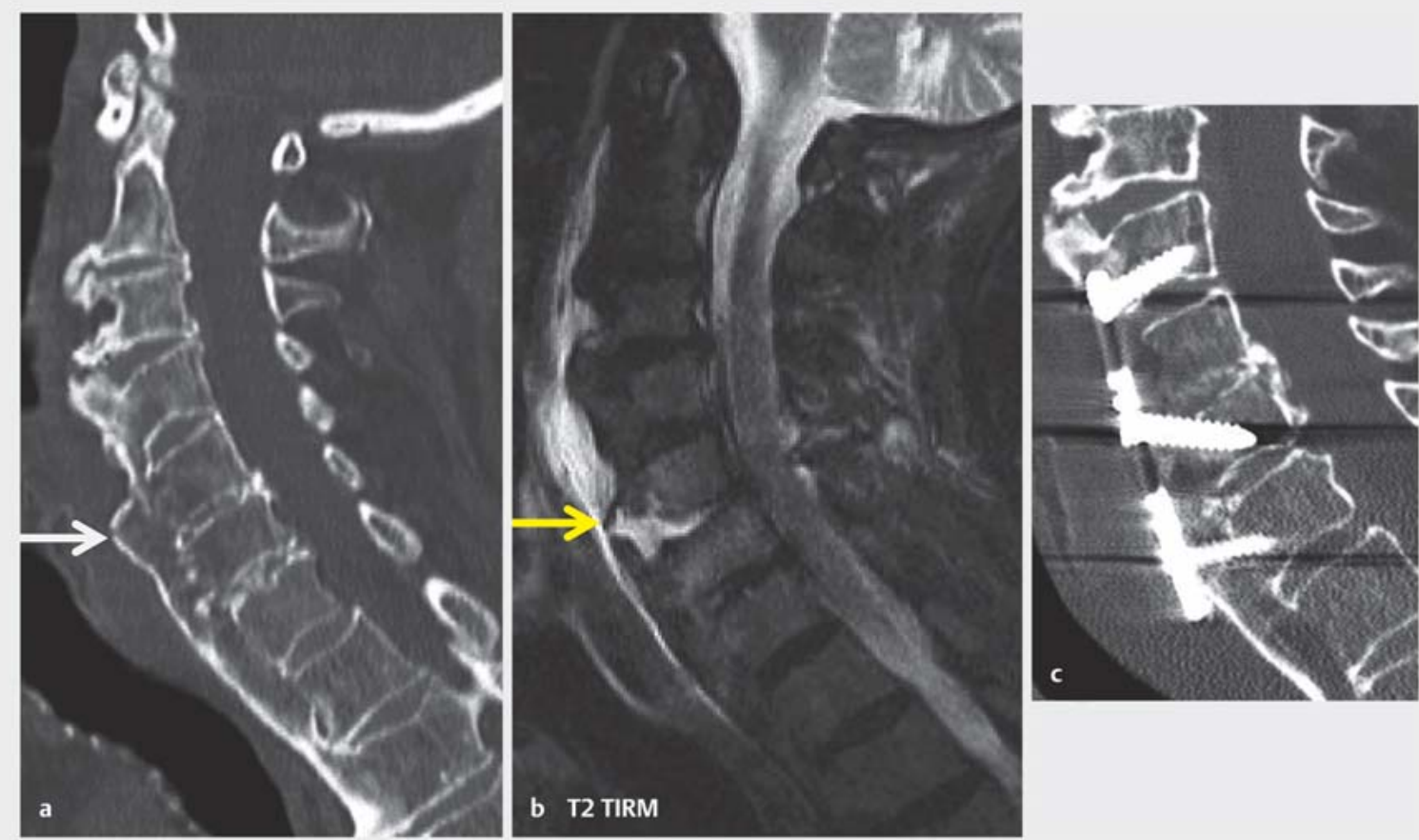

- Abb. 31 Transspondylophytäre-transdiskale Hyperextensionsfraktur HWK V/VI bei DISH (Röntgen negativ): a diskrete kortikale Kontinuitätsunterbechung (Pfeil). b MRT zeigt eine flüssigkeitsgefüllte, klaffende Lücke mit prävertebralem Hämatom (gelber Pfeil). c Z.n. ventraler Spondylodese. 


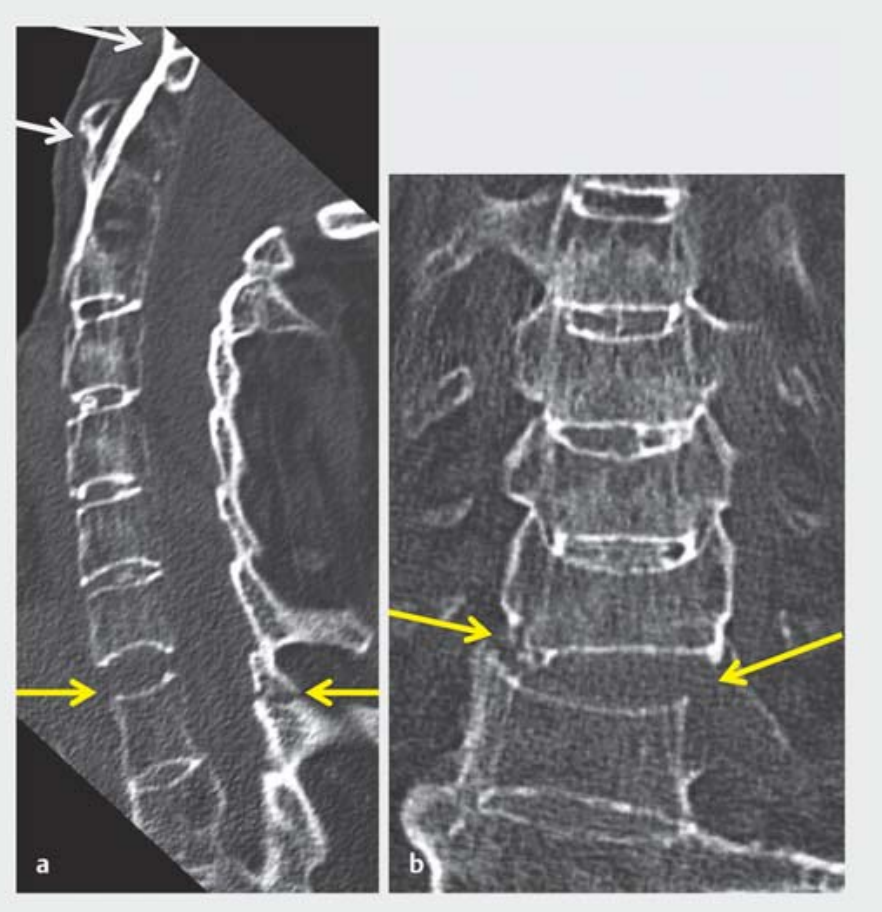

- Abb. 32 Transdiskale Fraktur bei Spondylarthropathia ankylopoetica Bechterew: vollständige diskoligamentäre Transsektion HWK VI/VII mit Horizontalfraktur durch den Bogen HWK VII (gelbe Pfeile in a). Die koronare Rekonstruktion zeigt die hochgradige Instabilität der Fraktur (gelbe Pfeile in b). Beachte die vordere kraniozervikale Ankylose (weiße Pfeile in a).

penköpfe bzw. -hälse (vgl. \ Abb. 7). Sie stellen starke pathomorphologische Hinweise auf einen wirksam gewordenen rotatorischen Kraftvektor dar, der wiederum höhergradige und schwerwiegendere Verletzungsmuster impliziert, einschließlich einer segmentalen Instabilität [57]. Diese Einschätzung gilt auch bei Abwesenheit von Rotationsfehlstellungen der HWS in der Akutphase (z. B. lagerungsbedingt im Stiff Neck).

\section{Ankylosierte Wirbelsäule (Rigid Spine)}

Ein besonderes und vielfach unterschätztes Problem stellt die traumatisch geschädigte, versteifte Halswirbelsäule dar [58]. Neben angeborenen oder erworbenen Blockwirbelbildungen, die meistens nur 1 oder 2 zervikale Segmente betreffen, sind es vor allem pontifizierende hyperostotische HWS-Veränderungen ( $\mathbf{A b b} \mathbf{3 1}$ ) oder die entzündlich bedingten Ankylosierungen (wie z. B. der Morbus Bechterew), die einerseits zu oft schwer zu identifizierenden Frakturen führen, andererseits aber auch hochgradig instabile transdiskale und transvertebrale Frakturen verursachen, die meistens nahezu horizontal das gesamte Wirbelsegment einschließlich der posterioren Elemente traversieren [59] ( $\bullet$ Abb. 32). Letzteres gilt in besonderem Maße für die Bechterew-erkrankte Wirbelsäule, die neben einer hochgradigen Osteoporose eine praktisch vollständig aufgehobene Schwingfähigkeit aufweist. Dies hat zur Folge, dass zum einen enorme Hebelkräfte an der Fraktur angreifen, was zu schweren Dislokationen führen kann mit der Folge deletärer Rückenmarksverletzungen [60], zum anderen sind spinale Etagenfrakturen nicht selten [61]: Die fehlende energieabsorbierende Schwingfähigkeit der ankylosierten Wirbelsäule kann zu distanten Frakturen am Achsenskelett führen, bei denen die zweite, entferntere Fraktur dann gewöhnlich übersehen wird. Klinisch schlecht beurteilbare Patienten oder ein unbekannter Traumamechanismus sollten daher in solchen Fällen zur radiologischen Abklärung der gesamten Wirbelsäule führen. Neben der hohen Rate neurologischer Komplikationen traumatisierter ankylosierter Wirbelsäulen [69] werden bei ihnen zudem auch häufiger epidurale Blutungskomplikationen gesehen ( $\bullet$ Abb. 33, [70]).

\section{Traumatische Bandscheibenextrusion}

Sie tritt in der Folge von Hyperextensions-Flexions-Verletzungen mit Zerreißung anulärer Bandstrukturen auf: Herniertes Bandscheibengewebe kann einserseits zur Myelonkompression unterschiedlichen Ausmaßes (bis hin zum diskogenen Querschnitt; [83]) führen, andererseits radikuläre Symptome hervorrufen [84]. Die Symptome treten oftmals verzögert zum Trauma auf [67]. Die MRT bietet gute Voraussetzungen, traumatische (mitunter sequestrierte) Bandscheibenextrusionen zu erkennen ( $\triangleright$ Abb. 34). Diese dürfen insbesondere vor geplanter geschlossener HWS-Reposition nicht übersehen werden (progressives neurologisches Defizit möglich; [68]). Mitunter werden diese traumatischen Bandscheibenvorfälle für epidurale Hämatome gehalten oder - vice versa - durch diese maskiert ( $\triangleright$ Abb. 35 ).

\section{Assoziierte Verletzungen}

- Myelonschäden: Diese reichen von Kontusionen über Quetschungen bis hin zu partiellen oder vollständigen Disruptionen mit den assoziierten klinischen Querschnittsymptomen [62,63]. Radikuläre Symptome kommen ebenfalls vor und werden vor allem im Zusammenhang mit zervikalen Plexusschäden beobachtet. Dabei kann das Myelon stippchenartige bis flächige Einblutungen aufweisen, die besonders gut auf T2* -gewichteten Sequenzen erkennbar sind [64]. Dabei ist darauf hinzuweisen, dass auch bei Erwachsenen signifikante Myelonschäden ohne radiologische Auffälligkeiten (z.B. Frakturen) angetroffen werden können [65].

- Hämatome: Epidurale Hämatome begleiten nicht selten Wirbelkörperfrakturen bzw. Verletzungen der dorsalen Zuggurtung und entstehen infolge traumatischer Läsionen der epiduralen Venenplexus ( $\mathbf{A} \mathbf{A b} \mathbf{b} . \mathbf{3 6}$ ) [66]. Ausgedehnte Blutansammlungen führen zu einer konsekutiven Thekalschlauchkompression mit Bedrängung des Myelons, was eine sofortige Entlastung erforderlich macht. Epidurale Hämatome können 

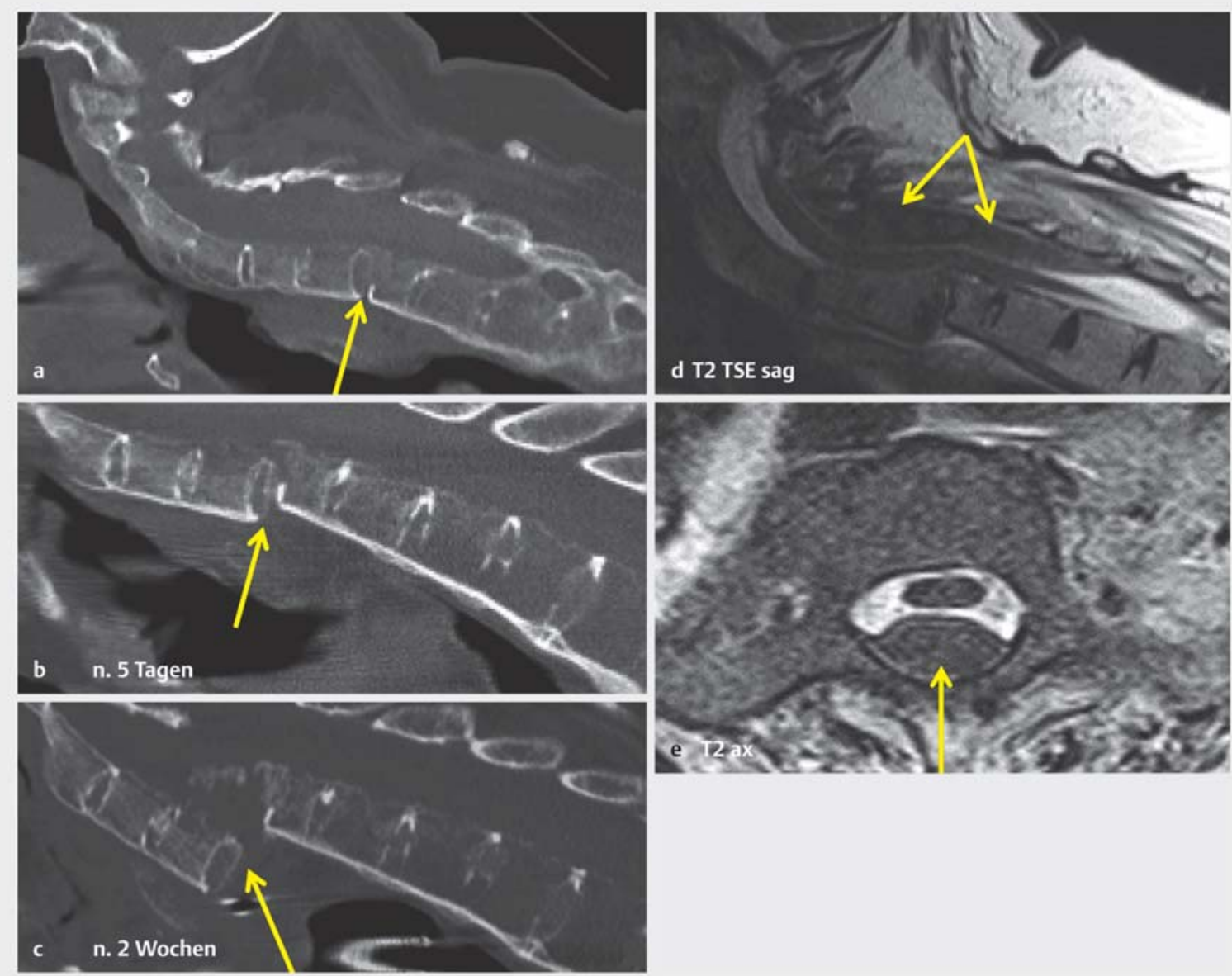

- Abb. 33 64-jähriger Patient, bekannter Morbus Bechterew, Leitersturz: a Initial „lediglich“ transdiskale Fraktur; später zunehmende (b) und hochgradige Dislokation (c) (Pfeile); d+e Ausgdehntes dorsales epidurales Hämatom (Pfeile).

gerade im HWS-Bereich leicht übersehen bzw. mit Liquorflussartefakten verwechselt werden, da - je nach Akuität und Alter der Hämatome - die Signalcharakteristik stark variiert und epidurales Fett zur besseren Hämatomabgrenzung im Zervikalbereich fehlt. In der CT lassen sich epidurale Hämatome nur sehr schlecht abgrenzen und werden demzufolge oft nicht erkannt. Ebenso sind sehr seltene atypische spinale Blutungslokalisationen (subdural, subarachnoidal) möglich und sollten beachtet werden ( $\bullet$ Abb. 37 ).

- Traumatische Vertebralisdissektionen: Sie kommen in ca. 0,5\% aller stumpfen kraniozervikalen Verletzungen (hauptsächlich Verkehrsunfälle) vor und betreffen vor allem jüngere Patienten [77]. Traumabedingte Scherkräfte verursachen Wandspannungen in den Vertebralarterien, die entweder zu Intimaeinrissen, intramuralen Hämatomen oder disseziierenden Aneurysmata führen (vgl. \ Abb. 13b) [78]. Das klinische Bild ist uneinheitlich, reicht von Nackenschmerz und Nackensteife bis hin zu schweren neurolog. Defiziten (Hirnstamminfarkte, Kleinhirninfarkte), oft verzögert auftretend. Das Problem besteht weniger in den mit-
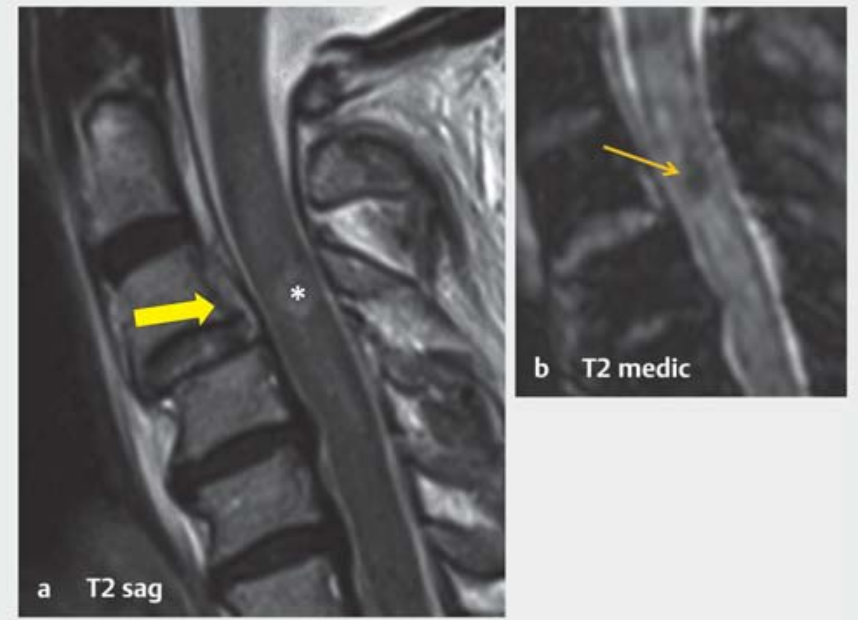

- Abb. 34 a Flammenartig nach kranial umgeschlagener subligamentärer (sequestrierter) Bandscheibenprolaps (Pfeil) mit Myelonkontusion. b Suszeptibilitätsartefakt infolge intramedullärer Hämorrhagie (Pfeil). 

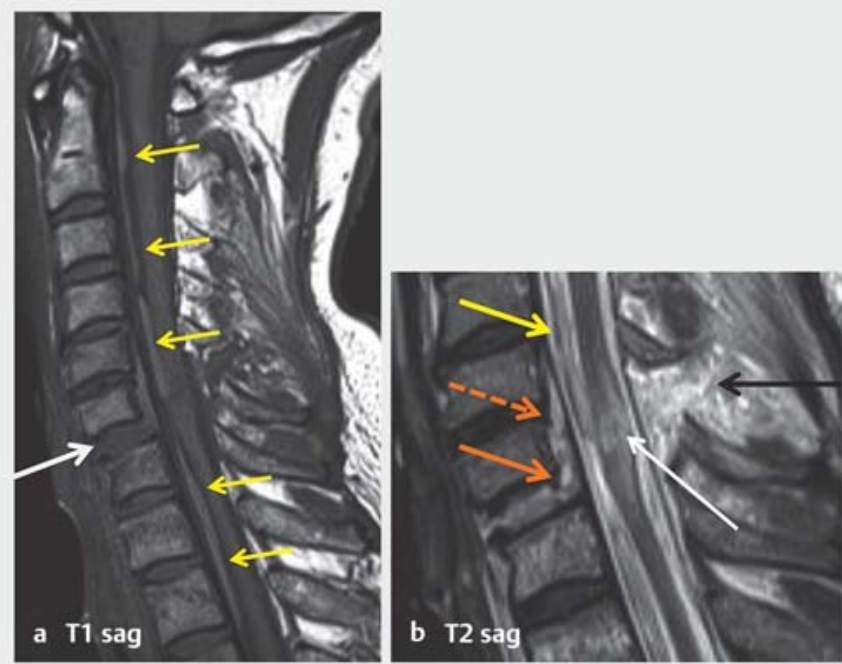

- Abb. 35 Verschiedene intraspinale Hämatomlokalisationen bei einer transdiskalen Luxationsfraktur HWK VI/VII (weißer Pfeil in a): a Langstreckiges segmentiertes subdurales Hämatom (gelbe Pfeile). b Traumatischer Bandscheibenprolaps (orangefarbener Pfeil) + schmales epidurales Hämatom (gestrichelter Pfeil) + subdurales Hämatom (gelber Pfeil); Myelonkontusion und -hämorrhagie (weißer Pfeil) und vollständige Ruptur der dorsalen Bänder (schwarzer Pfeil). unter nicht einfachen Nachweisbedingungen einer Vertebralisdissektion (CT-/MR-Angiografie, Duplexsonografie; [79]) als vielmehr darin, dass sie - vor allem bei Polytraumatisierten - zunächst wenig im klinischen Fokus stehen.

- Kettenfraktur: Nicht selten trifft man bei der MR-Abklärung zervikaler Verletzungen auf mehrere konsekutive, ventral betonte Deckplattenkontusionen bzw. flache Deckplattenimpressionen der oberen bis mittleren BWS, die dem CT-Nachweis wegen ihrer geringen Ausprägung oft entgehen; zumeist sind die BWK I-V betroffen. Sie entstehen durch einen kräftigen Flexionsimpuls auf die relativ starre obere BWS, wenn die - mehr oder weniger - mobile HWS peitschenartig nach vorn inkliniert wird ( $\bullet$ Abb. 38 ).

Einen Überblick über weitere zervikale Verletzungsmuster gibt $>$ Tab. 1:

\section{Wichtige Botschaften}

1. Der rationale Einsatz der Bildgebung an der HWS sollte sich nach kritischer Würdigung der klinischen Symptomatik an den etablierten Scores (CCSR, NEXUS) orientieren. Unter diesem Blickwinkel ist es wichtig zu wissen, welche diagnostische Aussage von der konventionellen Röntgendiagnostik erwartet werden kann und ob diese unter Beachtung des Schädigungs-

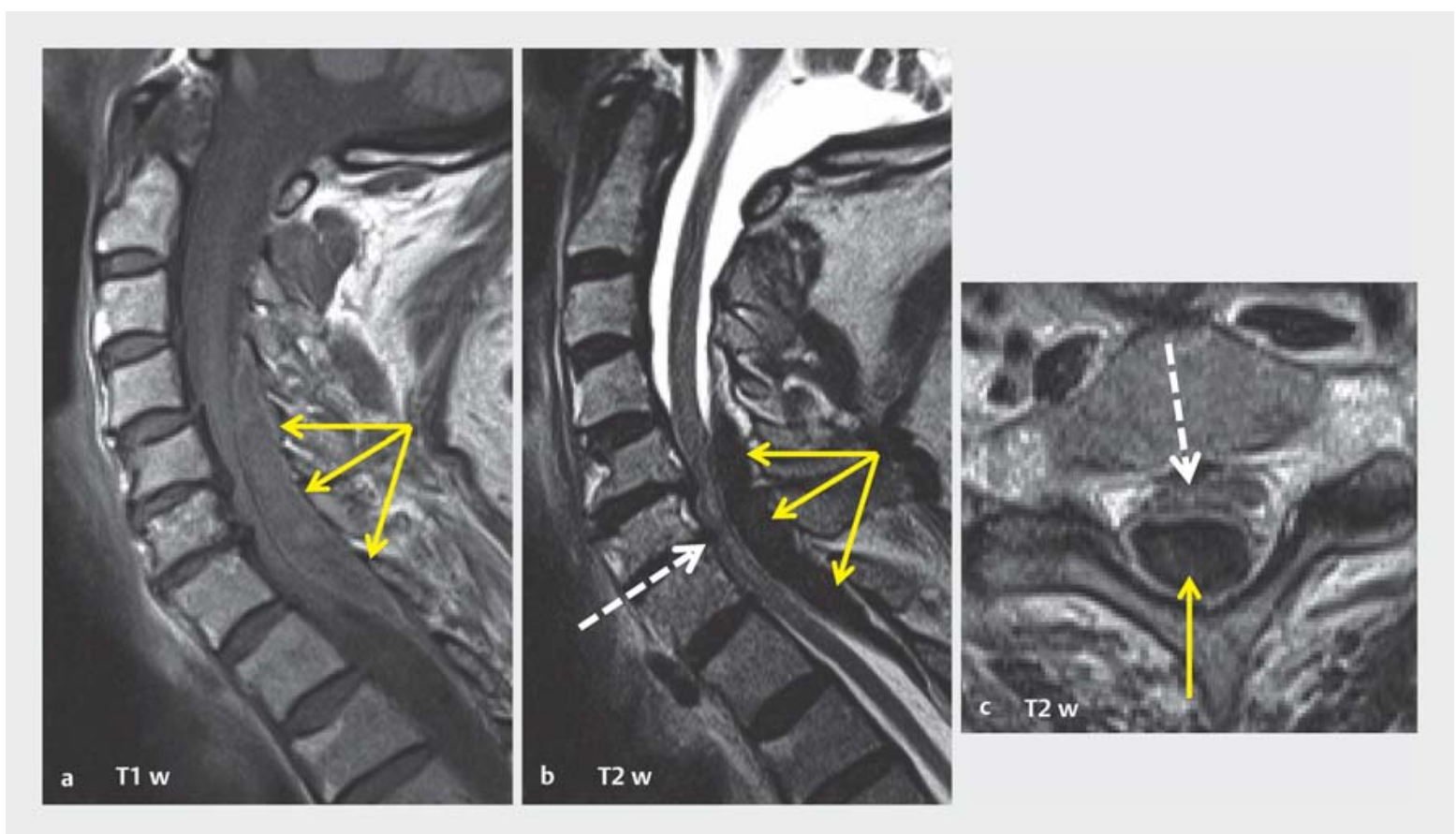

- Abb. 36 Ausgedehntes raumforderndes epidurales Hämatom (gelbe Pfeile in a-c). Beachte die deutliche Myelonkompression auf Höhe HWK V-BWK I (weiße Pfeile). Blut zeigt aufgrund zeitlich ablaufender Hämoglobindegradation unterschiedliche Signalcharakteristiken; im vorliegenden Fall handelt es sich um eine akute bis früh subakute Blutung. Quelle: Dr. C. Maurer, Freiburg. 


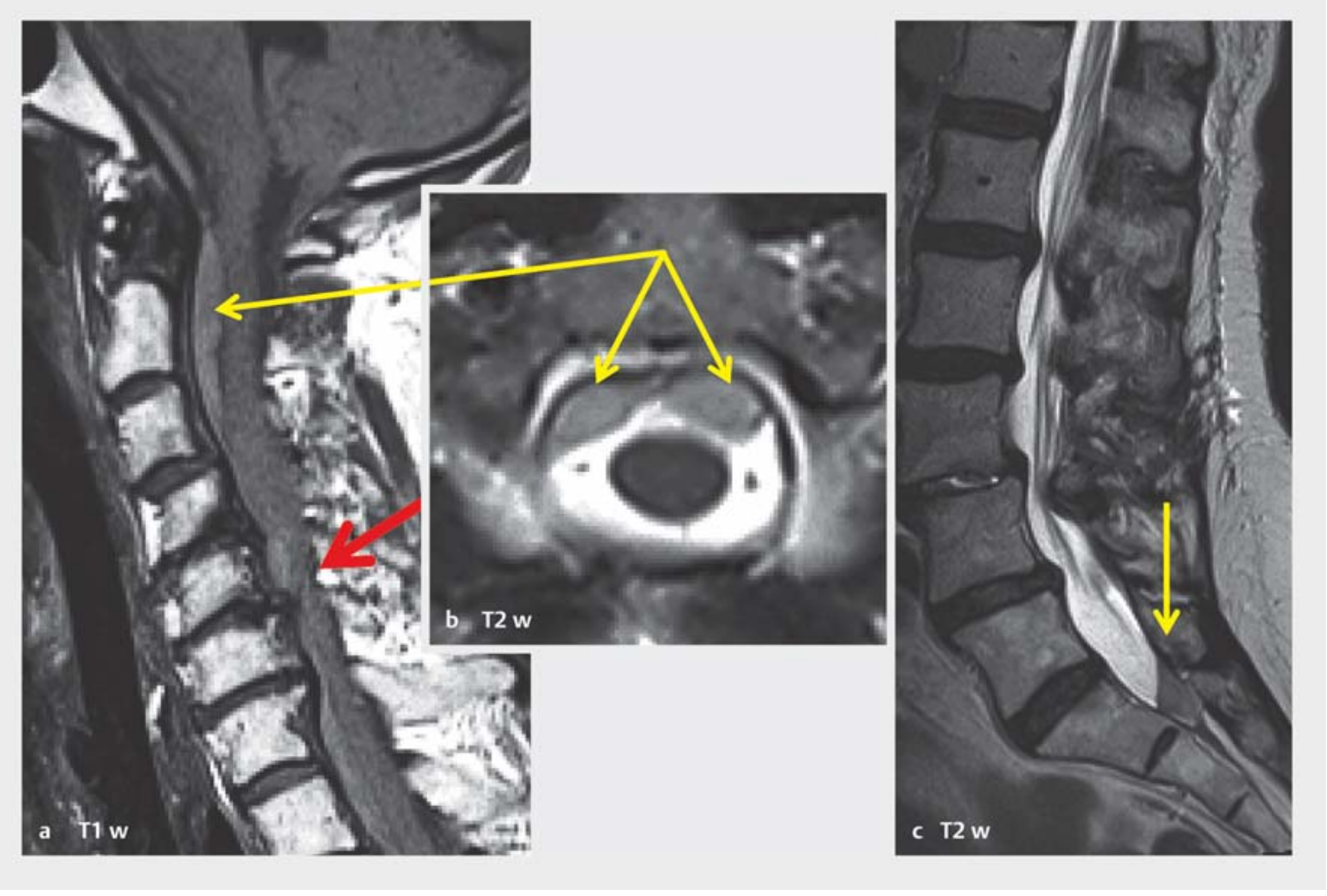

- Abb. 37 Spinales Subduralhämatom mit Subarachnoidalblutung (SAB): 75-jähriger Patient, Sturztrauma, progrediente Tetraparese: a Langstreckige signalintense subdurale Formation, vor allem auf Höhe C I/II (gelber Pfeil); beachte die degenerativ bedingte Spinalkanalstenose (roter Pfeil) als Ursache der Myelopathie (nicht gezeigt). b Kulissenartige subdurale Hämatomformation; der Epiduralraum ist frei. c Subarachnoidale Blutspiegelbildung im Thekalsack (gelber Pfeil): Koinzidenz von subduralem und subarachoidalem Blut! (SAB).

- Tab. 1 Übersicht besonderer und typischer subaxialer HWS-Verletzungen.

\begin{tabular}{|l|l|}
\hline Muster & Beschreibung \\
\hline Teardrop-Fraktur & dreieckförmiges Vorderunterkantenfragment (Absprengung bei Hyperflexion, Avulsion bei Hyperextension) \\
\hline Facet Interlocking & einseitig verhakte Facettengelenksluxation (stabile Blockierung; cave: Rotationsmechanismus!) \\
\hline Vertebralisdissektion & Folge starker Rotations-/ Distraktionskräfte mit Gefäßwandeinrissen; meist geringe bzW. verzögerte Symptomatik \\
\hline traumatische Diskusextrusion & dorsaler Anulusriss mit traumatischer (oft subligamentärer) Nucleus-pulposus-Hernierung \\
\hline „Kettenfraktur“ & $\begin{array}{l}\text { konsekutive ventrale Deckplattenimpressionsfrakturen der oberen BWS bei hyperflektierenden HWS-Verlet- } \\
\text { zungsmechanismen }\end{array}$ \\
\hline Schipperfraktur & $\begin{array}{l}\text { isolierte Dornfortsatzfraktur, zumeist HWK VII; kann durch direktes Trauma geschehen (Abbruchfraktur) oder } \\
\text { durch (repetitive) Stresseinwirkung (sog. „Kohleschipperfraktur“): Abrissfraktur }\end{array}$ \\
\hline
\end{tabular}

musters auch suffizient wiedergegeben wird (Problem falsch negativer Befunde in der Projektionsradiografie).

2. Relevante Traumen mit einem hohen Schädigungspotenzial bedürfen einheitlich einer modernen Multislice-CT-Abklärung. Ihre hohe diagnostische Qualität und überlagerungsfreie knöcherne Darstellung ermöglicht eine sichere Frakturdiagnostik und darüber hinaus auch eine indirekte Abschätzung grober diskoligamentärer Verletzungsmuster.

3. Der MRT kommt überragende Bedeutung bei der Darstellung diskoligamentärer Verletzungen, traumatischer Myelonläsionen und Bandscheibenextrusionen sowie intraspinaler Raumforderungen wie epidurale Hämatome zu. Vermutete versorgungspflichtige, instabile Läsionen, vor allem aber neurologische spinale Defizite, sollten stets zeitnah mittels MRT abgeklärt 


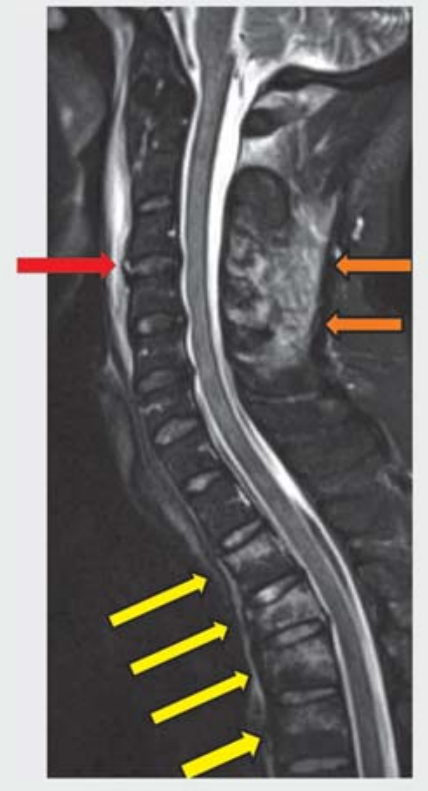

- Abb. 38 Junger Mann, Auffahrunfall: mögliche anteriore Anulusruptur (roter Pfeil) mit prävertebralem Hämatom; nuchales Weichteilödem ohne signifikante Bandruptur (orangefarbenene Pfeile); konsekutive, ventral betonte Kontusionen bzw. Impressionen der oberen BWS (gelbe Pfeile): sog. vertebrale Kettenfraktur (A1-Kompressionsfrakturen).

werden. Nur als Notfallmaßnahme kann ggf. ein Myelo-CT erfolgen, wenn ein MRT entweder nicht verfügbar oder nicht durchführbar ist (z. B. Schrittmacherpatient).

\section{Danksagung}

Der Autor dankt ausdrücklich Herrn Professor Dr. Dr. h.c. Edgar Mayr, Chefarzt der Klinik für Unfallchirurgie, Orthopädie, plastische und Handchirurgie des (Universitäts-) Klinikums Augsburg für die stets anregende und kritische Diskussion täglicher spinaler Verletzungsfälle zum wechselseitigen besseren Verständnis der oft komplizierten Schädigungsmuster.

Interessenkonflikt

Der Autor erklärt, dass kein Interessenkonflikt besteht.

\section{Autorinnen/Autoren}

\section{Thomas Grieser}

Dr., Klinik f. Diagnostische und Interventionelle Radiologie, Klinikum Augsburg

\section{Korrespondenzadresse}

\section{Dr. Thomas Grieser}

Klinik f. Diagnostische und Interventionelle Radiologie Klinikum Augsburg

Stenglinstr. 2

86156 Augsburg

Tel.: $0821 / 400-2441$

Fax: 0821/400-3312

Thomas.Grieser@klinikum-augsburg.de

\section{Literatur}

[1] Lowery DW, Wald MM, Browne BJ et al. Epidemiology of the cervical spine injury victims. Ann Emerg Med 2001; 38: 12-16

[2] Crim JR, Moore K, Brodke D. Clearance of the cervical spine in multitrauma patients: the role of advanced imaging. Semin Ultrasound CT MRI 2001; 22: 283-305

[3] Platzer P, Jaindl M, Thalhammer $G$ et al. Clearing the cervical spine in critically injured patients: a comprehensive C-spine protocol to avoid unnecessary delays in diagnosis. Eur Spine J 2006; 15: 1801-1810

[4] Rethnam U, Yesupalan RS, Bastawrous SS. The Swimmer's view: does it really show what it is supposed to show? A retrospective study. BMC Med Imaging 2008; 15: 2

[5] Walters BC, Hadley MN, Hurlbert RJ et al. Guidelines for the management of acute cervical spine and spinal cord injuries: 2013 Update. Neurosurgery 2013; 60 (Suppl. 1): S82-S91

[6] Hoffmann JR, Wolfson AB, Todd $K$ et al. Selective cervical spine radiography in blunt trauma: methodology of the National Emergency X-Radiography Utilization Study (NEXUS). Ann Emerg Med 1998; 32: 461-469

[7] Stiell IG, Wells GA, Vandemheen KL et al. The Canadian C-spine rule for radiography in alert and stable trauma patients. JAMA 2001; 286: 1841-1848

[8] Stiell IG, Clement CM, McKnight RD et al. The Canadian Cspine rule versus the NEXUS low-risk criteria in patients with trauma. N Engl J Med 2003; 349: 2510-2518

[9] Paykin G, O'Reilly G, Ackland HM et al. The NEXUS criteria are insufficient to exclude cervical spine fractures in older trauma patients. Injury 2017; 48: 1020-1024

[10] Chilvers G, Porter K, Choudhary S. Cervical spine clearance in adults following blunt trauma: a national survey across major trauma centres in England. Clin Radiol 2017; 12: 210-218

[11] Zakrison TL, Williams BH. Cervical spine evaluation in the blunt injured patient. Int J Surg 2016; 33: 246-250

[12] Raza M, Elkhodair S, Zaheer A et al. Safe cervical spine clearance in adult obtunded blunt trauma patients on the basis of a normal multidetector CT scan - a meta-analysis and cohort study. Injury 2013; 44: 1589-1595

[13] Panczykowski DM, Tomycz ND, Okonkwo DO. Comparative effectiveness of using computed tomography alone to exclude cervical spine injuries in obtunded or intubated patients: meta-analysis of 14,327 patients with blunt trauma. J Neurosurg 2011; 115: 541-549

[14] Müller E], Muhr G. Wirbelsäulenverletzungen. Stuttgart: Thieme; 1997; 66-77

[15] Daffner RH. Radiologische Diagnostik der Wirbelsäulenverletzungen. Stuttgart: Thieme; 1999: 199

[16] Hunter A, McGreevy J, Linden J. Pathologic C-spine fracture with low risk mechanism and normal physical exam. Am J Emerg Med 2017; 35: 1383.e1-1383.e2 
[17] Van Middendorp J], Albert T], Veth RP et al. Methodological systemic review: mortality in elderly patients with cervical spine injury: a critical appraisal of the reporting of baseline characteristics, follow-up, cause of death, and analysis of risk factors. Spine 2010; 35: 1079-1087

[18] Aarabi B, Mirvis S, Shanmuganathan K et al. Comparative effectiveness of surgical versus nonoperative management of unilateral, nondisplaced, subaxial cervical spine facet fractures without evidence of spinal cord injury: clinical article. J Neurosurg Spine 2014; 20: 270-277

[19] Sander AL, Laurer $H$, Lehnert T et al. A clinically useful classification of traumatic intervertebral disk lesions. AJR Am J Roentgenol 2013; 200: 618-623

[20] Morais DF, de Melo Neto JS, Meguins LC et al. Clinical applicability of magnetic resonance imaging in acute spinal cord trauma. Eur Spine J 2014; 23: 1457-1463

[21] Pizones J, Izquierdo E, Sanchez-Mariscal F et al. Sequential damage assessment of the different components of the posterior ligamentous complex after magnetic resonance imaging: prospective study 74 traumatic fractures. Spine (Phila $\mathrm{Pa}$ 1976) 2012; 37: E662-E667

[22] Maung AA, Johnson DC, Barre K et al. Cervical spine MRI in patients with negative CT: a prospective, multicenter study of the Research Consortium of New England Centers for Trauma (ReCONECT). J Trauma Acute Care Surg 2017; 82: 263-269

[23] Malhotra A, Wu X, Kalra VB et al. Utility of MRI for cervical spine clearance after blunt traumatic injury: a meta-analysis. Eur Radiol 2017; 27: 1148-1160

[24] Engelter ST, Grond-Grisbach C, Metso TM et al. Cervical artery dissection: trauma and other potential mechanical trigger events. Neurology 2013; 80: 1950-1957

[25] Haldeman S, Carey P, Townsend M et al. Clinical perceptions of the risk of vertebral artery dissection after cervical manipulation: the effect of referral bias. Spine J 2002; 2: 334-342

[26] Ringer AJ, Matern E, Parikh S et al. Screening for blunt cerebrovascular injury: selection criteria for use of angiography. J Neurosurg 2010; 112: 1146-1149

[27] White AA, Panjabi MM. The clinical biomechanics of the occipitoatlantoaxial complex. Orthop Clin North Am 1978; 9: 867878

[28] Anderson PA, Montesano PX. Morphology and treatment of occipital condyle fractures. Spine (Phila Pa 1976) 1988; 13 : 731-736

[29] Debernadi A, D'Aliberti G, Talamonti G et al. The craniovertebral junction area and the role of the ligaments and membranes. Neurosurgery 2015; 76 (Suppl. 1): S22-S32

[30] Tuli S, Tator CH, Fehlings MG et al. Occipital condyle fractures. Neurosurgery 1997; 41: 112-118

[31] Tan LA, Kasliwal MK, Traynelis VC. Comparison of CT and MRI findings for cervical spine clearance in obtunded patients without high impact trauma. Clin Neurol Neurosurg 2014; 120: 23-26

[32] Brown RL, Brunn MA, Garcia VF. Cervical spine injuries in children: a review of 103 patients treated consecutively at a level 1 pediatric trauma center. J Pediatr Surg 2001; 36: 1107-1114

[33] Carroll T, Smith CD, Liu X et al. Spinal cord injuries without radiologic abnormality in children: a systematic review. Spinal Cord 2015; 53: 842-848

[34] Matthiessen C, Robinson Y. Epidemiology of atlas fractures - a national registry-based cohort study of 1,537 cases. Spine J 2015; 15: 2332-2337

[35] Jefferson G. Fracture of the atlas vertebra. Report of four cases and a review of those previously recorded. Br J Surg 1919; 7: $407-422$
[36] Hadley MN, Dickman CA, Browner CM. Acute traumatic atlas fractures: management and long term outcome. Neurosurgery $1988 ; 23: 31-35$

[37] Goldberg W, Mueller C, Panacek E et al. Distribution and patterns of blunt traumatic cervical spine injury. Ann Emerg Med 2001; 38: 17-21

[38] Saternus K-S, Kernbach-Wighton G. Understanding cervical spinal Trauma: Biomechanics and Pathophysiology In: CassarPullicino VN, Imhof H, eds. Spinal Trauma - An Imaging Approach. Stuttgart: Thieme; 2006: 22-23

[39] Anderson LD, D'Alonzo RT. Fractures of the odontoid process of the axis. J Bone Joint Surg Am 1974; 56: 1663-1674

[40] Korres DS, Chytas DG, Markatos KN et al. The "challenging" fractures of the odontoid process: a review of the classification schemes. Eur J Orthop Surg Traumatol 2017; 27: 469-475

[41] Rozzelle C], Aarabi B, Dhall SS et al. Os odontoideum. Neurosurgery 2013; 72 (Suppl. 2): S159-S169

[42] Yurube T, Sumi M, Nishida K et al. Accelerated development of cervical spine instabilities in rheumatoid arthritis: a prospective minimum 5-year cohort study. PLoS One 2014; 9: e88970

[43] Joaquim AF, Ghizoni E, Tedeschi $\mathrm{H}$ et al. Radiological evaluation of cervical spine involvement in rheumatoid arthritis. Neurosurg Focus 2015; 38: E4

[44] Gleizes V, Jacquot FP, Signoret F et al. Combined injuries in the upper cervical spine: clinical and epidemiological data over a 14-year period. Eur Spine J 2000; 9: 386-392

[45] Schleicher P, Scholz M, Pingel A et al. Traumatic spondylolisthesis of the axis vertebra in adults. Global Spine J 2015; 5 : 346-358

[46] Rampersaud YR, Bederman SS. Management of C2 traumatic Spondylolisthesis (Hangman's Fracture) and other Variants. In: Spine and spinal Cord Trauma. Vaccaro AR, Fehlings MG, Dvorak MF, eds. New York: Thieme 2011; 248-262

[47] Kowalski HM, Cohen WA, Cooper P et al. Pitfalls in the CT diagnosis of atlantoaxial rotary subluxation. AJR Am J Roentgenol 1987; 149: 595-600

[48] Fielding JW, Hawkins RJ. Atlanto-axial rotatory fixation. J Bone Joint Surg Am 1977; 59: 37-44

[49] Greenbaum J, Walters N, Levy PD. An evidenced-based approach to radiographic assessment of cervical spine injuries in the emergency department. J Emerg Med 2009; 36: 64-71

[50] Vaccaro AR, Hulbert RJ, Patel AA et al. The subaxial cervical spine injury classification system: a novel approach to recognize the importance of morphology, neurology, and integrity of the disco-ligamentous complex. Spine (Phila Pa 1976) 2007; 32: 2365-2374

[51] Favero KJ, Van Peteghem PK. The quadrangular fragment fracture. Roentgenographic features and treatment protocol. Clin Orthop Relat Res 1989; (239): 40-46

[52] Saternus K-S, Kernbach-Wighton G. Understanding cervical spinal Trauma: Biomechanics and Pathophysiology. In: Spinal Trauma - An Imaging Approach. Cassar-Pullicino VN, Imhof H, eds. Stuttgart: Thieme; 2006: 24-34

[53] Korres D, Benetos I, Evangelopoulos D et al. Tear-drop fractures of the lower cervical spine: classification and analysis of 54 cases. Eur J Orthop Surg Traumatol 2007; 17: 521-526

[54] Vaccaro AR, Madigan L, Schweitzer ME et al. Magnetic resonance imaging analysis of soft tissue disruption after flexiondistraction injuries of the subaxial cervical spine. Spine (Phila Pa 1976) 2001; 26: 1866-1872

[55] Daffner RH, Verma SV. The significant signs of cervical vertebral trauma: a reassessment. Appl Radiol 1995; 24: 31-35

[56] Palmieri F, Cassar-Pullicino VN, Dell'Atti C et al. Uncovertebral joint injury in cervical facet dislocation: the headphones sign. Eur Radiol 2006; 16: 1312-1315 
[57] Shanmuganathan K, Mirvis SE, Levine AM. Rotational injury of cervical facets: CT analysis of fracture patterns with implications for management and neurologic outcome. AJR Am J Roentgenol 1994; 63: 1165-1169

[58] Faterni G, Gensler LS, Learch T] et al. Spine fractures in ankylosing spondylitis: a case report and review of imaging as well as predisposing factors to falls and fractures. Semin Arthritis Rheum 2014; 44: 20-24

[59] Olerud C, Frost A, Bring J. Spinal fractures in patients with ankylosing spondylitis. Eur Spine J 1996; 5: 51-55

[60] Teunissen FR, Verbeek BM, Cha TD et al. Spinal cord injury after traumatic spine fracture in patients with ankylosing spinal disorders. J Neurosurg Spine 2017; 27: 709-716

[61] Samartzis D, Anderson DG, Shen FH. Multiple and simultaneous spine fractures in ankylosing spondylitis: case report. Spine (Phila Pa 1976) 2005; 30: E711-E715

[62] Molligaj G, Payer M, Schaller K et al. Acute traumatic central cord syndrome: a comprehensive review. Neurochirurgie 2014; 60: 5-11

[63] De Smet E, Vanhoenacker FM, Parizel PM. Traumatic myelopathy: current concepts in imaging. Semin Musculoskeletal Radiol 2014; 18: 318-331

[64] Chandra J, Sheerin F, Lopez de Heredia L et al. MRI in acute and subacute post-traumatic spinal cord injury: pictorial review. Spinal Cord 2012; 50: 2-7

[65] Boese CK, Lechler P. Spinal cord injury without radiologic abnormalities in adults: a systematic review. J Trauma Acute Care Surg 2013; 75: 320-330

[66] Kumar Y, Hayashi D. Role of magnetic resonance imaging in acute spinal trauma: a pictorial review. BMC Musculoskeletal Disord 2016; 17: 310

[67] Pratt ES, Green DA, Spengler DM. Herniated intervertebral discs associated with unstable spinal injuries. Spine 1990; 15 : 662-666

[68] Vaccaro AR, Falatyn SP, Flanders AE et al. Magnetic resonance evaluation of the intervertebral disc, spinal ligaments, and spinal cord before and after closed traction reduction of cervical spine dislocations. Spine (Phila Pa 1976) 1999; 24: 1210-1217

[69] Westerveld LA, Verlaan J], Oner FC. Spinal fractures in patients with ankylosing spinal disorders: a systematic review of the literature on treatment, neurological status and complications. Eur Spine J 2009; 18: 145-156

[70] Elgafy H, Bransford RJ, Chapman JR. Epidural hematoma associated with occult fracture in ankylosing spondylitis patient: a case report and review of the literature. J Spine Disord Tech 2011; 24: 469-473

[71] Daffner RH, Harris JH. Cervical Spine Injuries. 5th ed. Philadelphia, PA; Lippincott Williams \& Wilkins; 2013: 139-245

[72] Vialle LR, Oner FC, Vaccaro AR, eds. AOSpine Masters Series. Vol. 5: Cervical Spine Trauma. Stuttgart: Thieme; 2015; 83146

[73] Ryken TC, Hadley MN, Walters BC et al. Radiographic assessment. Neurosurgery 2013; 72 (Suppl. 2): S54-S72

[74] Harris JH, Carson GC, Wagner LK. Radiologic diagnosis of traumatic occipitovertebral dissociation: 1. Normal occipitovertebral relationships on lateral radiograph of supine subjects. AJR Am J Roentgenol 1994; 162: 881-886.

[75] Harris JH, Carson GC, Wagner LK et al. Radiologic diagnosis of traumatic occipitovertebral dissociation: 2. Comparison of three methods of detecting occipitovertebral relationships on lateral radiograph of supine subjects. AJR Am J Roentgenol 1994; 162: 887-892

[76] Wang S, Wang C, Yan M et al. Novel surgical classification and treatment strategy for atlantoaxial dislocations. Spine (Phila Pa 1976) 2013; 38: E1348-E1356
[77] Nedeltchev K, Baumgartner RW. Traumatic cervical artery dissection. Dront Neurol Neurosci 2005; 20: 54-63

[78] Mohan IV. Current optimal assessment and management of carotid and vertebral spontaneous and traumatic dissection. Angiology 2014; 65: 274-283

[79] Gottesman RF, Sharma P, Robinson KA et al. Imaging characteristics of symptomatic vertebral artery dissection: a systematic review. Neurologist 2012; 18: 255-260

[80] Joaquim AF, Patel AA, Vaccaro AR. Cervical injuries scored according to the subaxial injury classification system: an analysis of the literature. J Craniovertebr Junction Spine 2014; 5: 65-70

[81] Stone AT, Bransford RJ, Lee MJ et al. Reliability of classification systems for subaxial cervical injuries. Evid Based Spine Care 2010; 1: 19-26

[82] Joaquim AF, Ghizoni E, Tedeschi $\mathrm{H}$ et al. Clinical results of patients with subaxial cervical spine trauma treated according to the SLIC score. J Spinal Cord Med 2014; 37: 420-424

[83] Dai ], Jia L. Central cord injury complicating acute cervical disc herniation in trauma. Spine (Phila Pa 1976) 2000; 25: 331-335

[84] Farmer J, Vaccaro A, Albert T] et al. Neurologic deterioration after cervical spine cord injury. J Spinal Disorders 1998; 11: 192-196

[85] Stiell IG, Wells GA Vandemheen KL et al. The Canadian C-spine rule for radiography in alert and stable trauma patients. JAMA 2001; 286: 1841-1848

[86] Dickman CA, Greene KA, Sonntag VK. Injuries involving the transverse atlantal ligament: classification and treatment guidelines based upon experience with 39 injuries. Neurosurgery 1996; 38: 44-50

[87] Traynelis VC, Marano GD, Dunker RD et al. Traumatic atlantooccipital dislocation. Case report. J Neurosurg 1986; 65: 863870

[88] Horn EM, Feiz-Erfan I, Lekovic GP et al. Survivors of occipitoatlantal dislocation injuries: imaging and clinical correlates. J Neurosurg Spine 2007; 6: 113-120

[89] Jackson RS, Banit DM, Rhyme AL III et al. Upper cervical spine injuries. J Am Acad Orthop Surg 2002; 10: 271-280

[90] Gehweiler ], Duff D, Martinez S et al. Fractures of the atlas vertebra. Skeletal Radiol 1976; 1: 97-102

[91] Landells CD, Van Peteghem PK. Fractures of the atlas: classification, treatment and morbidity. Spine (Phila Pa 1976) 1998; 13: 450-452

[92] Roy-Camille R, Saillant G, Judet T et al. [Factors of severity in the fractures of the odontoid process]. Rev Chir Orthop Repar Appar Mot 1980; 66: 183-186

[93] Effendi B, Roy D, Cornish B et al. Fractures of the ring of the axis. A classification based on the analysis of 131 cases. J Bone Joint Surg Br 1981; 63-B: 313-327

[94] Levine AM, Edwards CC. The management of traumatic spondylolisthesis of the axis. J Bone Joint Surg Am 1985; 67: 217226

[95] Powers B, Miller MD, Kramer RS et al. Traumatic anterior atlanto-occipital dislocation. Neurosurgery 1979; 4: 12-17

Bibliografie

DOI https://doi.org/10.1055/a-0586-5241

OP-JOURNAL 2018; 34: 88-110 (c) Georg Thieme Verlag KG Stuttgart · New York ISSN 0178-1715 